\title{
Quantum phase transitions of antiferromagnets and the cuprate superconductors
}

\author{
Subir Sachdev
}

\begin{abstract}
I begin with a proposed global phase diagram of the cuprate superconductors as a function of carrier concentration, magnetic field, and temperature, and highlight its connection to numerous recent experiments. The phase diagram is then used as a point of departure for a pedagogical review of various quantum phases and phase transitions of insulators, superconductors, and metals. The bond operator method is used to describe the transition of dimerized antiferromagnetic insulators between magnetically ordered states and spin-gap states. The Schwinger boson method is applied to frustrated square lattice antiferromagnets: phase diagrams containing collinear and spirally ordered magnetic states, $Z_{2}$ spin liquids, and valence bond solids are presented, and described by an effective gauge theory of spinons. Insights from these theories of insulators are then applied to a variety of symmetry breaking transitions in $d$-wave superconductors. The latter systems also contain fermionic quasiparticles with a massless Dirac spectrum, and their influence on the order parameter fluctuations and quantum criticality is carefully discussed. I conclude with an introduction to strong coupling problems associated with symmetry breaking transitions in two-dimensional metals, where the order parameter fluctuations couple to a gapless line of fermionic excitations along the Fermi surface.
\end{abstract}

\section{Introduction}

The cuprate superconductors have stimulated a great deal of innovative theoretical work on correlated electron systems. On the experimental side, new experimental techniques continue to be discovered and refined, leading to striking advances over twenty years after the original discovery of high temperature superconductivity [1].

Subir Sachdev

Department of Physics, Harvard University, Cambridge MA 02138, USA.

e-mail: sachdevephysics.harvard.edu 


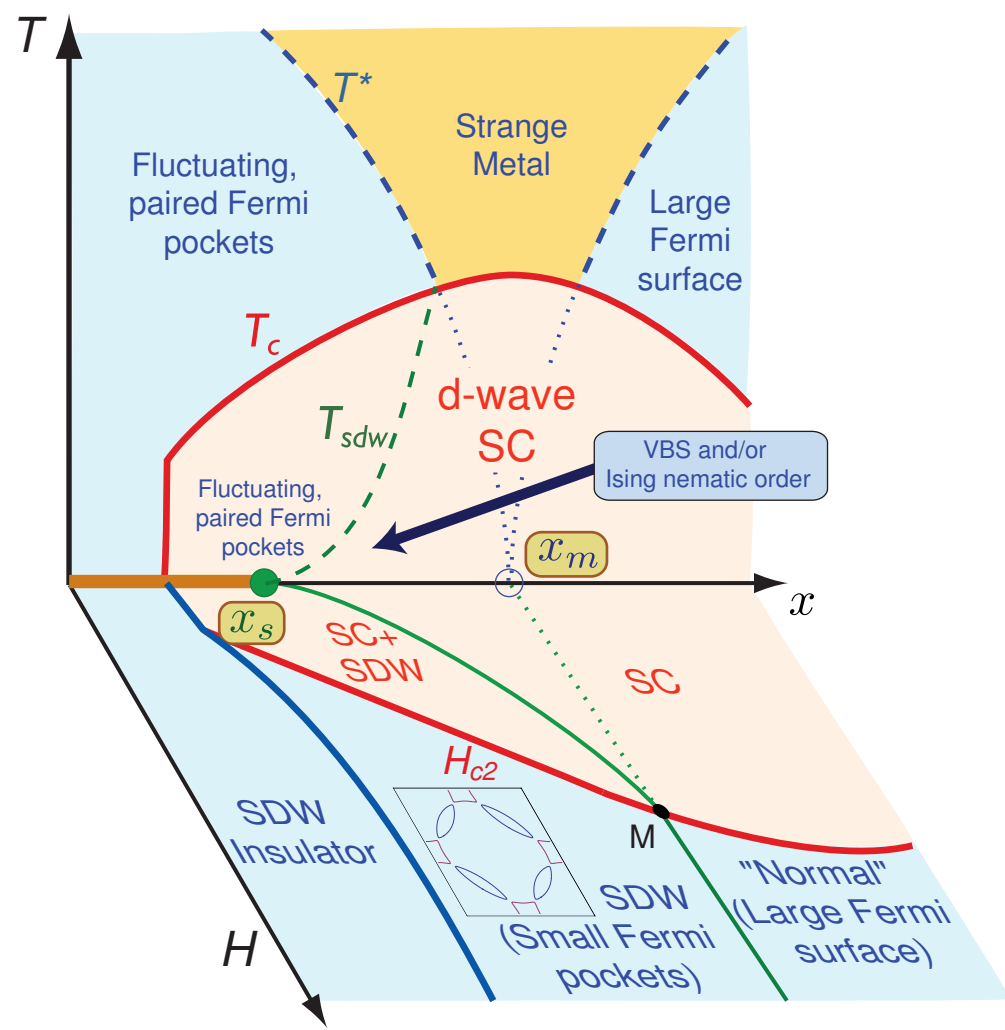

Fig. 1 Proposed global phase diagram for the hole- and electron-doped cuprates $|3-5|$. The axes are carrier concentration $(x)$, temperature $(T)$, and a magnetic field $(H)$ applied perpendicular to the square lattice. The regions with the SC label have $d$-wave superconductivity. The strange metal and the "pseudogap" regime are separated by the temperature $T^{*}$. Dashed lines indicate crossovers. After accounting for the valence bond solid (VBS) or Ising nematic orders that can appear in the regime $x_{s}<x<x_{m}$, the dashed $T^{*}$ line and the dotted line connecting $x_{m}$ to the point $M$ become true phase transitions. There can also be fractionalized phases in the region $x_{s}<x<x_{m}$ , as discussed recently in Refs. [6.7].

In the past few years, a number of experiments, and most especially the discovery of quantum oscillations in the underdoped regime [2], have shed remarkable new light on the origins of cuprate superconductivity. I believe these new experiments point to a synthesis of various theoretical ideas, and that a global theory of the rich cuprate phenomenology may finally be emerging. The ingredients for this synthesis were described in Refs. [3-5], and are encapsulated in the phase diagram shown in Fig. 1. Here I will only highlight a few important features of this phase diagram, and use those as motivations for the theoretical models described in these lectures. The reader is referred to the earlier papers [4] 5] for a full discussion of the experimental support for these ideas. Throughout the lectures, I will refer back to Fig. 1] and point 
out the relevance of various field theories to different aspects of this rich phase diagram.

It is simplest to examine the structure of Fig. 1 beginning from the regime of large doping. There, ample evidence has established that the ground state is a conventional Fermi liquid, with a single "large" Fermi surface enclosing the area demanded by the Luttinger theorem. Because of the underlying band structure, this large Fermi surface is hole-like (for both electron and hole-doping), and so encloses an area $1+x$ for hole density $x$, and an area $1-p$ for doped electron density $p$. The central quantum phase transition (QPT) in Fig. 1 1 is the onset of spin density wave (SDW) order in this large Fermi surface metal at carrier concentration $x=x_{m}$, shown in Fig. 1 near the region where $T_{c}$ is largest (the subscript $m$ refers to the fact that the transition takes place in a metal); we will describe this transition in more detail in Section 4. Because of the onset of superconductivity, the QPT at $x=x_{m}$ is revealed only at magnetic fields strong enough to suppress superconductivity, i.e., at $H>H_{c 2}$. For $x<x_{m}$, we then have a Fermi liquid metal with SDW order. Close to the transition, when the SDW order is weak, the large Fermi surface is generically broken up by the SDW order into "small" electron and hole pockets, each enclosing an area of order $x$ (see Fig. 17 later in the text). Note that electron pockets are present both for hole and electron doping: such electron pockets in the hole-doped cuprates were first discussed in Ref. [8]. There is now convincing experimental evidence for the small Fermi pockets in the hole underdoped cuprates, including accumulating evidence for electron pockets [9, 10]. The QPT between the small and large Fermi surface metals is believed to be at $x_{m} \approx 0.24$ in the hole-doped cuprates [11,12], and at $p_{m} \approx 0.165$ in the electron-doped cuprates [13 14]. One of the central claims of Fig. 1 1 is that it is the QPT at $x=x_{m}$ which controls the non-Fermi liquid "strange metal" behavior in the normal state above the superconductivity $T_{c}$. We leave open the possibility [15] that there is an extended non-Fermi liquid phase for a range of densities with $x>x_{m}$ : this is not shown in Fig. 1. and will be discussed here only in passing.

The onset of superconductivity near the SDW ordering transition of a metal has been considered in numerous previous works [16,17]. These early works begin with the large Fermi surface found for $x>x_{m}$, and consider pairing induced by exchange of SDW fluctuations; for the cuprate Fermi surface geometry, they find an attractive interaction in the $d$-wave channel, leading to $d$-wave superconductivity. Because the pairing strength is proportional to the SDW fluctuations, and the latter increase as $x \searrow x_{m}$, we expect $T_{c}$ to increase as $x$ is decreased for $x>x_{m}$, as is shown in Fig. 1 Thus for $x>x_{m}$, stronger SDW fluctuations imply stronger superconductivity, and the orders effectively attract each other.

It was argued in Ref. [3] that the situation becomes qualitatively different for $x<$ $x_{m}$. This becomes clear from an examination of Fig. 1 as a function of decreasing $T$ for $x<x_{m}$. It is proposed [3, 18] in the figure that the Fermi surface already breaks apart locally into the small pocket Fermi surfaces for $T<T^{*}$. So the onset of superconductivity at $T_{c}$ involves the pairing of these small Fermi surfaces, unlike the large Fermi surface pairing considered above for $x>x_{m}$. For $x<x_{m}$, an increase in local SDW ordering is not conducive to stronger superconductivity: the 
SDW order 'eats up' the Fermi surface, leaving less room for the Cooper pairing instability on the Fermi surface. Thus in this regime we find a competition between SDW ordering and superconductivity for 'real estate' on the Fermi surface [3, 19]. As we expect the SDW ordering to increase as $x$ is decreased for $x<x_{m}$, we should have a decrease in $T_{c}$ with decreasing $x$, as is indicated in Fig. 1]

We are now ready to describe the second important feature of Fig. 1. The complement of the suppression of superconductivity by SDW ordering is the suppression of SDW ordering by superconductivity. The competition between superconductivity and SDW order moves [3] the actual SDW onset at $H=0$ and $T=0$ to a lower carrier concentration $x=x_{s}$ (or $p=p_{s}$ for electron doping). The QPT at $x=x_{s}$ controls the criticality of spin fluctuations within the superconducting phase (and hence the subscript $s$ ), while that $x=x_{m}$ continues to be important for $T>T_{c}$ (as is indicated in Fig. 1). There is now a line of SDW-onset transitions within the superconducting phase [20], connecting the point $x_{s}$ to the point $M$, for which there is substantial experimental evidence [21-26]. The magnitude of the shift from $x_{m}$ to $x_{s}$ depends a great deal upon the particular cuprate: it is largest in the materials with the strongest superconductivity and the highest $T_{c}$. In the hole-doped YBCO series we estimate $x_{s} \approx 0.085$ [26] and in the hole-doped LSCO series we have $x_{s} \approx 0.14$ [23] (recall our earlier estimate $x_{m} \approx 0.24$ in the hole-doped cuprates [11, 12]), while in the electron-doped cuprate $\mathrm{Nd}_{2-x} \mathrm{Ce}_{x} \mathrm{CuO}_{4}$, we have $p_{s}=0.145$ [27] (recall $p_{m} \approx 0.165$ in the electron-doped cuprates [13. 14]).

With the shift in SDW ordering from $x_{m}$ to $x_{s}$, the need for the crossover line labeled $T_{\mathrm{sdw}}$ in Fig. 1 1 becomes evident. This is the temperature at which the electrons finally realize that they are to the 'disordered' of the actual SDW ordering transition at $x=x_{s}$, rather than to the 'ordered' side of the transition at $x=x_{m}$. Thus, for $T<T_{\text {sdw }}$, the large Fermi surface re-emerges at the lowest energy scales, and SDW order is never established. This leaves us with an interesting superconducting state at $T=0$, where the proximity to the Mott insulator can play an important role. Other orders linked to the antiferromagnetism of the Mott insulator can appear here, such as valence bond solid (VBS) and Ising-nematic order [28], or even topologically ordered phases [6,7]; experimental evidence for such orders has appeared in a number of recent experiments [12,29,-31], and we will study these orders in the sections below.

The shift in the SDW ordering from $x_{m}$ to $x_{s}$ has recently emerged as a generic property of quasi-two-dimensional correlated electron superconductors, and is not special to the cuprates. Knebel et al. [32] have presented a phase diagram for $\mathrm{CeRhIn}_{5}$ as a function of temperature, field, and pressure (which replaces carrier concentration) which is shown in Fig. 2 Notice the very similar structure to Fig. 1. the critical pressure for the onset of antiferromagnetism shifts from the metal to the superconductor, so that the range of antiferromagnetism is smaller in the superconducting state. In the pnictides, the striking observations by the Ames group [33-35] on $\mathrm{Ba}\left[\mathrm{Fe}_{1-x} \mathrm{Co}_{x}\right]_{2} \mathrm{As}_{2}$ show a 'back-bending' in the SDW onset temperature upon entering the superconducting phase: see Fig. 2. This is similar to the back-bending of the line $T_{\mathrm{sdw}}$ from $T^{*}$ in Fig. 1. and so can also be linked to the shift in the SDW onset transition between the metal and the superconductor. 

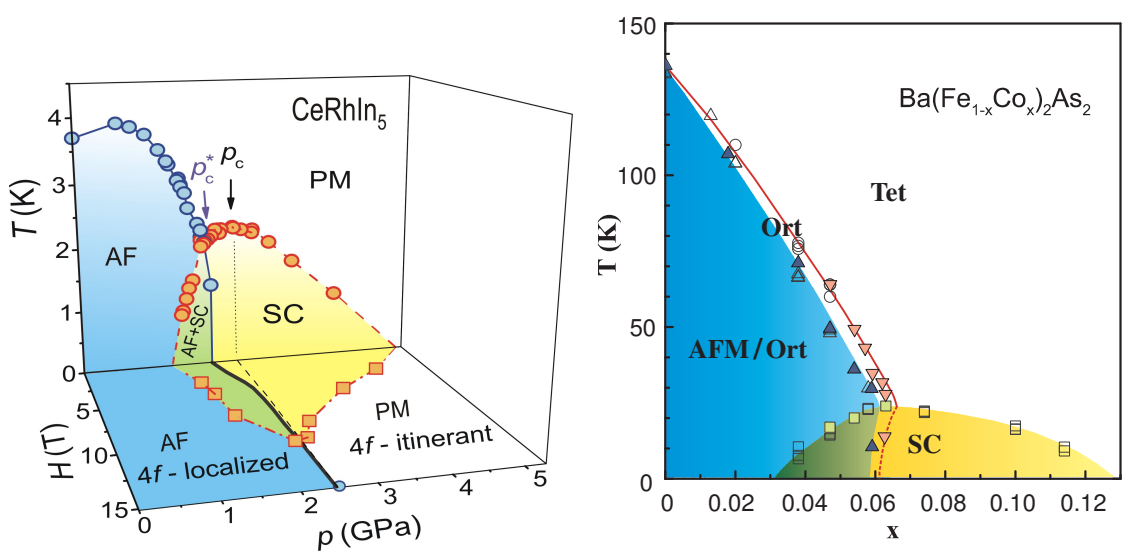

Fig. 2 Phase diagrams for CeRhIn 5 from Ref. [32] and for $\mathrm{Ba}\left[\mathrm{Fe}_{1-x} \mathrm{Co}_{x}\right]_{2} \mathrm{As}_{2}$ from Refs. 33 . 35]. For CeRhIn ${ }_{5}$, the shift from $p_{c}$ to $p_{c}^{*}$ is similar to the shift from $x_{m}$ to $x_{s}$ in Fig. 11 this shift is significantly larger in the cuprates (and especially in YBCO) because the superconductivity is stronger. In the ferropnictide $\mathrm{Ba}\left[\mathrm{Fe}_{1-x} \mathrm{Co}_{x}\right]_{2} \mathrm{As}_{2}$, the back-bending of the SDW ordering transition in the superconducting phase is similar to that of $T_{\mathrm{sdw}}$ in Fig. 1]

It is clear from Fig. 2 that the shift in the SDW order between the metal and the superconductor is relatively small in the non-cuprate materials, and may be overlooked in an initial study without serious consequences. Similar comments apply to the electron-doped cuprates. However, the shift is quite large in the hole-doped cuprates: this can initially suggest that the cuprates are a different class of materials, with SDW ordering playing a minor role in the physics of the superconductivity. One of the main claims of Fig. 1 is that after accounting for the larger shift in the SDW transition, all of the cuprates fall into a much wider class of correlated electron superconductors for which the SDW ordering transition in the metal is the central QPT controlling the entire phase diagram (see also the recent discussion by Scalapino [36]).

Our discussion will be divided into 3 sections, dealing with the nature of quantum fluctuations near SDW ordering in insulators, $d$-wave superconductors, and metals respectively. These cases are classified according to the increasing density of states for single-electron excitations. We will begin in Section 2 by considering a variety of Mott insulators, and describe their phase diagrams. The results will apply directly to experiments on insulators not part of the cuprate family. However, we will also gain insights, which will eventually be applied to various aspects of Fig. 1 for the cuprates. Then we will turn in Section 3 to $d$-wave superconductors, which have a Dirac spectrum of single-electron excitations as described in Section 3.1. Their influence on the SDW ordering transition at $x=x_{s}$ will be described using field-theoretical methods in Section 3.2. Section 3.3 will describe the Ising-nematic ordering at or near $x=x_{s}$ indicated in Fig. 1 . Finally, in Section 4 , we will turn to metals, which have a Fermi surface of low-energy single-particle excitations. We will summarize the current status of QPTs of metals: in two dimensions most QPTs 
lead to strong coupling problems which have not been conquered. It is clear from Fig. 1 that such QPTs are of vital importance to the physics of metallic states near $x=x_{m}$.

Significant portions of the discussion in the sections below have been adapted from other review articles by the author [38-40].

\section{Insulators}

The insulating state of the cuprates at $x=0$ is a $S=1 / 2$ square lattice antiferromagnet, which is known to have long-range Néel order. We now wish to study various routes by which quantum fluctuations may destroy the Néel order. In this section, we will do this by working with undoped insulators in which we modify the exchange interactions. These do not precisely map to any of the transitions in the phase diagram in Fig. 11, but we will see in the subsequent sections that closely related theories do play an important role.

The following subsections will discuss two distinct routes to the destruction of Néel order in two-dimensional antiferromagnet. In Section 2.1 we describe coupled dimer antiferromagnets, in which the lattice has a natural dimerized structure, with $2 S=1 / 2$ spins per unit cell which can pair with each other. These are directly relevant to experiments on materials like $\mathrm{TlCuCl}_{3}$. We will show that these antiferromagnets can be efficiently described by a bond-operator method. Then in section 2.2 we will consider the far more complicated and subtle case where the lattice has full square lattice symmetry with only a single $S=1 / 2$ spin per unit cell, and the Néel order is disrupted by frustrating exchange interactions. We will explore the phase diagram of such antiferromagnets using the Schwinger boson method. These results have direct application to experimental and numerical studies of a variety of two-dimensional Mott insulators on the square, triangular, and kagome lattices; such applications have been comprehensively reviewed in another recent article by the author [37], and so will not be repeated here.

\subsection{Coupled dimer antiferromagnets: bond operators}

We consider the "coupled dimer" Hamiltonian [41]

$$
H_{d}=J \sum_{\langle i j\rangle \in \mathcal{A}} \boldsymbol{S}_{i} \cdot \boldsymbol{S}_{j}+g J \sum_{\langle i j\rangle \in \mathcal{B}} \boldsymbol{S}_{i} \cdot \boldsymbol{S}_{j}
$$

where $\boldsymbol{S}_{j}$ are spin-1/2 operators on the sites of the coupled-ladder lattice shown in Fig. 33 with the $\mathcal{A}$ links forming decoupled dimers while the $\mathcal{B}$ links couple the dimers as shown. The ground state of $H_{d}$ depends only on the dimensionless coupling $g$, and we will describe the low temperature $(T)$ properties as a function of $g$. 


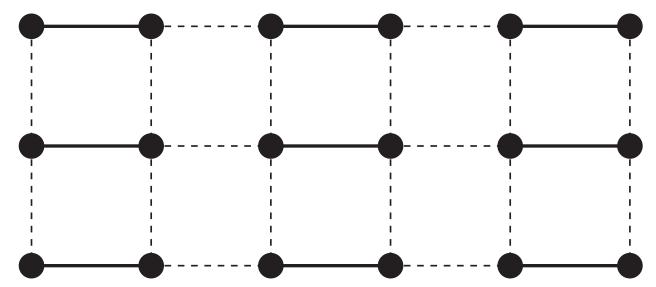

Fig. 3 The coupled dimer antiferromagnet. Spins $(S=1 / 2)$ are placed on the sites, the $\mathcal{A}$ links are shown as full lines, and the $\mathcal{B}$ links as dashed lines.

We will restrict our attention to $J>0$ and $0 \leq g \leq 1$. A three-dimensional model with the same structure as $H_{d}$ describes the insulator $\mathrm{TlCuCl}_{3}[42-44]$.

Note that exactly at $g=1, H_{d}$ is identical to the square lattice antiferromagnet, and this is the only point at which the Hamiltonian has only one spin per unit cell. At all other values of $g, H_{d}$ has a pair of $S=1 / 2$ spins in each unit cell of the lattice.

\subsubsection{Phases and their excitations}

Let us first consider the case where $g$ is close to 1 . Exactly at $g=1, H_{d}$ is identical to the square lattice Heisenberg antiferromagnet, and this is known to have longrange, magnetic Néel order in its ground state, i.e., the spin-rotation symmetry is broken and the spins have a non-zero, staggered, expectation value in the ground state with

$$
\left\langle\boldsymbol{S}_{j}\right\rangle=\eta_{j} N_{0} \boldsymbol{n},
$$

where $\boldsymbol{n}$ is some fixed unit vector in spin space, $\eta_{j}$ is \pm 1 on the two sublattices, and $N_{0}$ is the Néel order parameter. This long-range order is expected to be preserved for a finite range of $g$ close to 1 . The low-lying excitations above the ground state consist of slow spatial deformations in the orientation $\boldsymbol{n}$ : these are the familiar spin waves, and they can carry arbitrarily low energy, i.e., the phase is 'gapless'. The spectrum of the spin waves can be obtained from a text-book analysis of small fluctuations about the ordered Néel state using the Holstein-Primakoff method [45]: such an analysis yields two polarizations of spin waves at each wavevector $k=\left(k_{x}, k_{y}\right)$ (measured from the antiferromagnetic ordering wavevector), and they have excitation energy $\varepsilon_{k}=\left(c_{x}^{2} k_{x}^{2}+c_{y}^{2} k_{y}^{2}\right)^{1 / 2}$, with $c_{x}, c_{y}$ the spin-wave velocities in the two spatial directions.

Let us turn now to the vicinity of $g=0$. Exactly at $g=0, H_{d}$ is the Hamiltonian of a set of decoupled dimers, with the simple exact ground state wavefunction shown in Fig. 4, the spins in each dimer pair into valence bond singlets, leading to a paramagnetic state which preserves spin rotation invariance and all lattice symmetries. Excitations are now formed by breaking a valence bond, which leads to a three-fold degenerate state with total spin $S=1$, as shown in Fig. 5 k. At $g=0$, this broken bond is localized, but at finite $g$ it can hop from site-to-site, leading to 


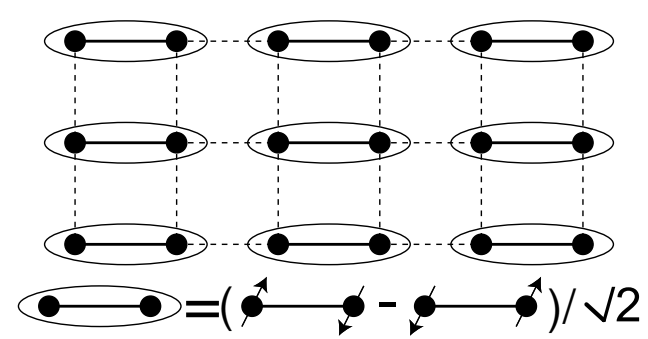

Fig. 4 Schematic of the quantum paramagnet ground state for small $g$. The ovals represent singlet valence bond pairs.

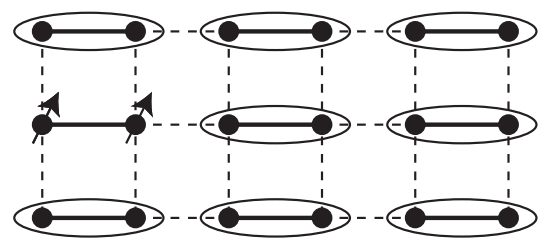

(a)

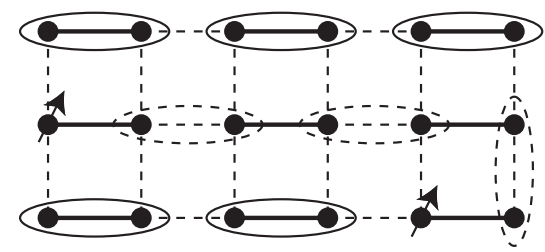

(b)

Fig. 5 (a) Cartoon picture of the bosonic $S=1$ excitation of the paramagnet. (b) Fission of the $S=1$ excitation into two $S=1 / 2$ spinons. The spinons are connected by a "string" of valence bonds (denoted by dashed ovals) which lie on weaker bonds; this string costs a finite energy per unit length and leads to the confinement of spinons.

a triplet quasiparticle excitation. Note that this quasiparticle is not a spin-wave (or equivalently, a 'magnon') but is more properly referred to as a spin 1 exciton or a triplon. We parameterize its energy at small wavevectors $k$ (measured from the minimum of the spectrum in the Brillouin zone) by

$$
\varepsilon_{k}=\Delta+\frac{c_{x}^{2} k_{x}^{2}+c_{y}^{2} k_{y}^{2}}{2 \Delta},
$$

where $\Delta$ is the spin gap, and $c_{x}, c_{y}$ are velocities; we will provide an explicit derivation of (3) in Section 2.1.2 Fig. 5 also presents a simple argument which shows that the $S=1$ exciton cannot fission into two $S=1 / 2$ 'spinons'.

The very distinct symmetry signatures of the ground states and excitations between $g \approx 1$ and $g \approx 0$ make it clear that the two limits cannot be continuously connected. It is known that there is an intermediate second-order phase transition at [41.46] $g=g_{c}=0.52337(3)$ between these states as shown in Fig. 6. Both the spin gap $\Delta$ and the Néel order parameter $N_{0}$ vanish continuously as $g_{c}$ is approached from either side. 


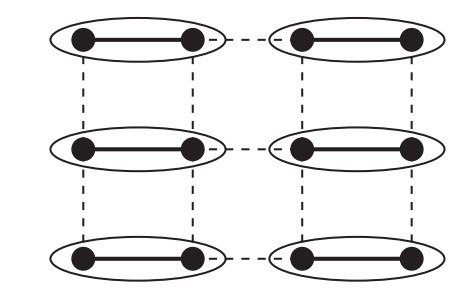

Paramagnet

Gapped triplon excitations

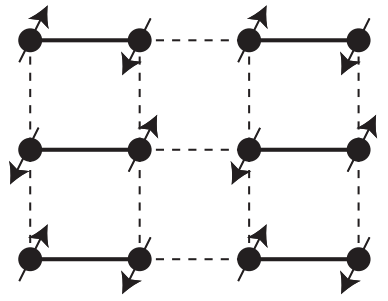

Neel

Spin wave excitations

$g_{c}$

Fig. 6 Ground states of $H_{d}$ as a function of $g$. The quantum critical point is at $|46| g_{c}=$ $0.52337(3)$. The compound $\mathrm{TlCuCl}_{3}$ undergoes a similar quantum phase transition under applied pressure [42 44].

\subsubsection{Bond operators and quantum field theory}

In this section we will develop a continuum description of the low energy excitations in the vicinity of the critical point postulated above. There are a number of ways to obtain the same final theory: here we will use the method of bond operators [47 48], which has the advantage of making the connection to the lattice degrees of freedom most direct. We rewrite the Hamiltonian using bosonic operators which reside on the centers of the $\mathcal{A}$ links so that it is explicitly diagonal at $g=0$. There are 4 states on each $\mathcal{A} \operatorname{link}(|\uparrow \uparrow\rangle,|\uparrow \downarrow\rangle,|\downarrow \uparrow\rangle$, and $|\downarrow \downarrow\rangle)$ and we associate these with the canonical singlet boson $s$ and the canonical triplet bosons $t_{a}(a=x, y, z)$ so that

$$
\begin{aligned}
& |s\rangle \equiv s^{\dagger}|0\rangle=\frac{1}{\sqrt{2}}(|\uparrow \downarrow\rangle-|\downarrow \uparrow\rangle) ;\left|t_{x}\right\rangle \equiv t_{x}^{\dagger}|0\rangle=\frac{-1}{\sqrt{2}}(|\uparrow \uparrow\rangle-|\downarrow \downarrow\rangle) ; \\
& \left|t_{y}\right\rangle \equiv t_{y}^{\dagger}|0\rangle=\frac{i}{\sqrt{2}}(|\uparrow \uparrow\rangle+|\downarrow \downarrow\rangle) ;\left|t_{z}\right\rangle \equiv t_{z}^{\dagger}|0\rangle=\frac{1}{\sqrt{2}}(|\uparrow \downarrow\rangle+|\downarrow \uparrow\rangle) .
\end{aligned}
$$

Here $|0\rangle$ is some reference vacuum state which does not correspond to a physical state of the spin system. The physical states always have a single bond boson and so satisfy the constraint

$$
s^{\dagger} s+t_{a}^{\dagger} t_{a}=1 .
$$

By considering the various matrix elements $\left\langle s\left|\boldsymbol{S}_{1}\right| t_{a}\right\rangle,\left\langle s\left|\boldsymbol{S}_{2}\right| t_{a}\right\rangle, \ldots$, of the spin operators $\boldsymbol{S}_{1,2}$ on the ends of the link, it follows that the action of $\boldsymbol{S}_{1}$ and $\boldsymbol{S}_{2}$ on the singlet and triplet states is equivalent to the operator identities

$$
S_{1 a}=\frac{1}{2}\left(s^{\dagger} t_{a}+t_{a}^{\dagger} s-i \epsilon_{a b c} t_{b}^{\dagger} t_{c}\right),
$$




$$
S_{2 a}=\frac{1}{2}\left(-s^{\dagger} t_{a}-t_{a}^{\dagger} s-i \epsilon_{a b c} t_{b}^{\dagger} t_{c}\right)
$$

where $a, b, c$ take the values $x, y, z$, repeated indices are summed over and $\epsilon$ is the totally antisymmetric tensor. Inserting (6) into (1), and using (5), we find the following Hamiltonian for the bond bosons:

$$
\begin{aligned}
& H_{d}=H_{0}+H_{1} \\
& H_{0}=J \sum_{\ell \in \mathcal{A}}\left(-\frac{3}{4} s_{\ell}^{\dagger} s_{\ell}+\frac{1}{4} t_{\ell a}^{\dagger} t_{\ell a}\right) \\
& H_{1}=g J \sum_{\ell, m \in \mathcal{A}}\left[a(\ell, m)\left(t_{\ell a}^{\dagger} t_{m a} s_{m}^{\dagger} s_{\ell}+t_{\ell a}^{\dagger} t_{m a}^{\dagger} s_{m} s_{\ell}+\text { H.c. }\right)+b(\ell, m)\right. \\
& \left.\times\left(i \epsilon_{a b c} t_{m a}^{\dagger} t_{\ell b}^{\dagger} t_{\ell c} s_{m}+\text { H.c. }\right)+c(\ell, m)\left(t_{\ell a}^{\dagger} t_{m a}^{\dagger} t_{m b} t_{\ell b}-t_{\ell a}^{\dagger} t_{m b}^{\dagger} t_{m a} t_{\ell b}\right)\right],
\end{aligned}
$$

where $\ell, m$ label links in $\mathcal{A}$, and $a, b, c$ are numbers associated with the lattice couplings which we will not write out explicitly. Note that $H_{1}=0$ at $g=0$, and so the spectrum of the paramagnetic state is fully and exactly determined. The main advantage of the present approach is that application of the standard methods of many body theory to (7), while imposing the constraint (5), gives a very satisfactory description of the phases with $g \neq 0$, including across the transition to the Néel state. In particular, an important feature of the bond operator approach is that the simplest mean field theory already yields ground states and excitations with the correct quantum numbers; so a strong fluctuation analysis is not needed to capture the proper physics.

A complete numerical analysis of the properties of (7) in a self-consistent Hartree-Fock treatment of the four boson terms in $H_{1}$ has been presented in Ref. [47]. In all phases the $s$ boson is well condensed at zero momentum, and the important physics can be easily understood by examining the structure of the lowenergy action for the $t_{a}$ bosons. For the particular Hamiltonian (1), the spectrum of the $t_{a}$ bosons has a minimum at the momentum $(0, \pi)$, and for large enough $g$ the $t_{a}$ condense at this wavevector: the representation (6) shows that this condensed state is the expected Néel state, with the magnetic moment oscillating as in (2). The condensation transition of the $t_{a}$ is therefore the quantum phase transition between the paramagnetic and Néel phases of the coupled dimer antiferromagnet. In the vicinity of this critical point, we can expand the $t_{a}$ bose field in gradients away from the $(0, \pi)$ wavevector: so we parameterize

$$
t_{\ell, a}(\tau)=t_{a}\left(r_{\ell}, \tau\right) e^{i(0, \pi) \cdot r_{\ell}}
$$

where $\tau$ is imaginary time, $\boldsymbol{r} \equiv(x, y)$ is a continuum spatial coordinate, and expand the effective action in spatial gradients. In this manner we obtain 


$$
\begin{gathered}
\mathcal{S}_{t}=\int d^{2} r d \tau\left[t_{a}^{\dagger} \frac{\partial t_{a}}{\partial \tau}+C t_{a}^{\dagger} t_{a}-\frac{D}{2}\left(t_{a} t_{a}+\text { H.c. }\right)+K_{1 x}\left|\partial_{x} t_{a}\right|^{2}+K_{1 y}\left|\partial_{y} t_{a}\right|^{2}\right. \\
\left.+\frac{1}{2}\left(K_{2 x}\left(\partial_{x} t_{a}\right)^{2}+K_{2 y}\left(\partial_{y} t_{a}\right)^{2}+\text { H.c. }\right)+\cdots\right]
\end{gathered}
$$

Here $C, D, K_{1,2 x, y}$ are constants that are determined by the solution of the selfconsistent equations, and the ellipses represent terms quartic in the $t_{a}$. The action $\mathcal{S}_{t}$ can be easily diagonalized, and we obtain a $S=1$ quasiparticle excitation with the spectrum

$$
\varepsilon_{k}=\left[\left(C+K_{1 x} k_{x}^{2}+K_{1 y} k_{y}^{2}\right)^{2}-\left(D+K_{2 x} k_{x}^{2}+K_{2 y} k_{y}^{2}\right)^{2}\right]^{1 / 2} .
$$

This is, of course, the triplon (or spin exciton) excitation of the paramagnetic phase postulated earlier in (3); the latter result is obtained by expanding (10) in momenta, with $\Delta=\sqrt{C^{2}-D^{2}}$. This value of $\Delta$ shows that the ground state is paramagnetic as long as $C>D$, and the quantum critical point to the Néel state is at $C=D$.

The critical point and the Néel state are more conveniently described by an alternative formulation of $\mathcal{S}_{t}$ (although an analysis using bond operators directly is also possible [49]). It is useful to decompose the complex field $t_{a}$ into its real and imaginary parts as follows

$$
t_{a}=Z\left(\varphi_{a}+i \pi_{a}\right),
$$

where $Z$ is a normalization chosen below. From $(8)$ and the connection to the lattice spin operators, it is not difficult to show that the vector $\varphi_{a}$ is proportional to the Néel order parameter $\boldsymbol{n}$ in Eq. (2). Insertion of (11) into (9) shows that the field $\pi_{a}$ has a quadratic term $\sim(C+D) \pi_{a}^{2}$, and so the coefficient of $\pi_{a}^{2}$ remains large even as the spin gap $\Delta$ becomes small. Consequently, we can safely integrate $\pi_{a}$ out, and the resulting action for the Néel order parameter $\varphi_{a}$ takes the form

$$
\mathcal{S}_{\varphi}=\int d^{2} r d \tau\left[\frac{1}{2}\left\{\left(\partial_{\tau} \varphi_{a}\right)^{2}+c_{x}^{2}\left(\partial_{x} \varphi_{a}\right)^{2}+c_{y}^{2}\left(\partial_{y} \varphi_{a}\right)^{2}+s \varphi_{a}^{2}\right\}+\frac{u}{24}\left(\varphi_{a}^{2}\right)^{2}\right] .
$$

Here we have chosen $Z$ to fix the coefficient of the temporal gradient term, and $s=C^{2}-D^{2}$.

The action $\mathcal{S}_{\varphi}$ gives a simple picture of excitations across the quantum critical point, which can be quantitatively compared to neutron scattering experiments [44] on $\mathrm{TlCuCl}_{3}$. In the paramagnetic phase $(s>0)$, a triplet of gapped excitations is observed, corresponding to the three normal modes of $\varphi_{a}$ oscillating about $\varphi_{a}=0$; as expected, this triplet gap vanishes upon approaching the quantum critical point. In a mean field analysis, the field theory in Eq. 12 has a triplet gap of $\sqrt{s}$ (mean field theory is applicable to $\mathrm{TlCuCl}_{3}$ because this antiferromagnet is three dimensional). In the Néel phase, the neutron scattering detects 2 gapless spin waves, and one gapped longitudinal mode [50] (the gap to this longitudinal mode vanishes at the quantum critical point), as is expected from fluctuations in the inverted 'Mexican hat' potential of $\mathcal{S}_{\varphi}$ for $s<0$. The longitudinal mode has a mean-field energy 


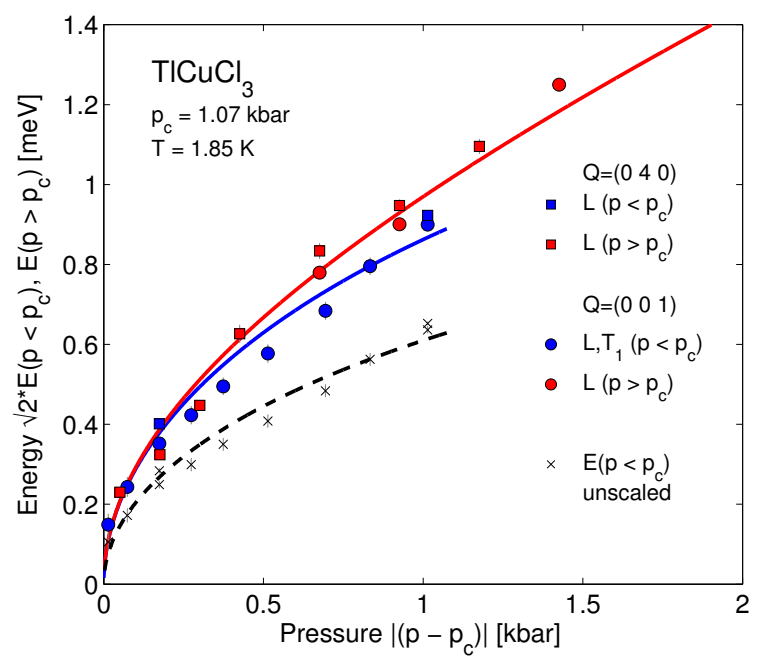

Fig. 7 Energies of the gapped collective modes across the pressure $(p)$ tuned quantum phase transition in $\mathrm{TlCuCl}_{3}$ observed by Ruegg et al. [44]. We test the description by the action $\mathcal{S}_{\varphi}$ in Eq. [12] with $s \propto\left(p_{c}-p\right)$ by comparing $\sqrt{2}$ times the energy gap for $p<p_{c}$ with the energy of the longitudinal mode for $p>p_{c}$. The lines are the fits to a $\sqrt{\left|p-p_{c}\right|}$ dependence, testing the $1 / 2$ exponent.

gap of $\sqrt{2|s|}$. These mean field predictions for the energy of the gapped modes on the two sides of the transition are tested in Fig. 7 the observations are in good agreement with the $1 / 2$ exponent and the predicted $[37,51] \sqrt{2}$ ratio, providing a non-trival experimental test of the $\mathcal{S}_{\varphi}$ field theory.

We close this subsection by noting that all of the above results have a direct generalization to other lattices. One important difference that emerges in such calculations on some frustrated lattices [52] is worth noting explicitly here: the minimum of the $t_{a}$ spectrum need not be at special wavevector like $(0, \pi)$, but can be at a more generic wavevector $\boldsymbol{K}$ such that $\boldsymbol{K}$ and $-\boldsymbol{K}$ are not separated by a reciprocal lattice vector. A simple example which we consider here is an extension of (1) in which there are additional exchange interactions along all diagonal bonds oriented "northeast' (so that the lattice has the connectivity of a triangular lattice). In such cases, the structure of the low energy action is different, as is the nature of the magnetically ordered state. The parameterization (8) must be replaced by

$$
t_{\ell a}(\tau)=t_{1 a}\left(r_{\ell}, \tau\right) e^{i \boldsymbol{K} \cdot \boldsymbol{r}_{\ell}}+t_{2 a}\left(r_{\ell}, \tau\right) e^{-i \boldsymbol{K} \cdot \boldsymbol{r}_{\ell}},
$$

where $t_{1,2 a}$ are independent complex fields. Proceeding as above, we find that the low-energy effective action 12 is replaced by

$$
\mathcal{S}_{\Phi}=\int d^{2} r d \tau\left[\left|\partial_{\tau} \Phi_{a}\right|^{2}+c_{x}^{2}\left|\partial_{x} \Phi_{a}\right|^{2}+c_{y}^{2}\left|\partial_{y} \Phi_{a}\right|^{2}+s\left|\Phi_{a}\right|^{2}\right.
$$




$$
\left.+\frac{u}{2}\left(\left|\Phi_{a}\right|^{2}\right)^{2}+\frac{v}{2}\left|\Phi_{a}^{2}\right|^{2}\right] .
$$

where now $\Phi_{a}$ is a complex field such that $\left\langle\Phi_{a}\right\rangle \sim\left\langle t_{1 a}\right\rangle \sim\left\langle t_{2 a}^{\dagger}\right\rangle$. Notice that there is now a second quartic term with coefficient $v$. If $v>0$, configurations with $\Phi_{a}^{2}=0$ are preferred: in such configurations $\Phi_{a}=n_{1 a}+i n_{2 a}$, where $n_{1,2 a}$ are two equal-length orthogonal vectors. Then from (13) and (6) it is easy to see that the physical spins possess spiral order in the magnetically ordered state in which $\Phi_{a}$ is condensed. For the case $v<0$, the optimum configuration has $\Phi_{a}=n_{a} e^{i \theta}$ where $n_{a}$ is a real vector: this leads to a magnetically ordered state with spins polarized collinearly in a spin density wave at the wavevector $\boldsymbol{K}$. The critical properties of the model in Eq. [14] have been described in Ref. [53].

\subsection{Frustrated square lattice antiferromagnets: Schwinger bosons}

As discussed at the beginning of Section 2, the more important and complex cases of quantum antiferromagnets are associated with those that have a single $S=1 / 2$ spin per unit cell. Such models are more likely to have phases in which the exotic spinon excitations of Fig. 5 are deconfined, i.e., their ground states possess neutral $S=1 / 2$ excitations and 'topological' order. We will meet the earliest established examples [54,55] of such phases below.

We are interested in Hamiltonians of the form

$$
\mathcal{H}=\sum_{i, j} J_{i j} \boldsymbol{S}_{i} \cdot \boldsymbol{S}_{j}
$$

where we consider the general case of $\boldsymbol{S}_{i}$ being spin $S$ quantum spin operators on the sites, $i$, of a 2-dimensional lattice. The $J_{i j}$ are short-ranged antiferromagnetic exchange interactions. We will mainly consider here the so-called square lattice $J_{1}$ $J_{2}-J_{3}$ model, which has first, second, and third neighbor interactions (see Fig. 8). Similar results have also been obtained on the triangular and kagome lattices [56. 57.

The main direct applications of the results here are to experiments on a variety of two-dimensional Mott insulators on the square, triangular, and kagome lattices. As noted earlier, we direct the reader to Ref. [37] for a discussion of these experiments. There have also been extensive numerical studies, also reviewed in the previous article [37], which are in good accord with the phase diagrams presented below. Applications to the cuprates, and to Fig. 1. will be discussed in the following sections.

A careful examination of the non-magnetic 'spin-liquid' phases requires an approach which is designed explicitly to be valid in a region well separated from Néel long range order, and preserves SU(2) symmetry at all stages. It should also be designed to naturally allow for neutral $S=1 / 2$ excitations. To this end, we introduce the Schwinger boson description [58], in terms of elementary $S=1 / 2$ bosons. For 


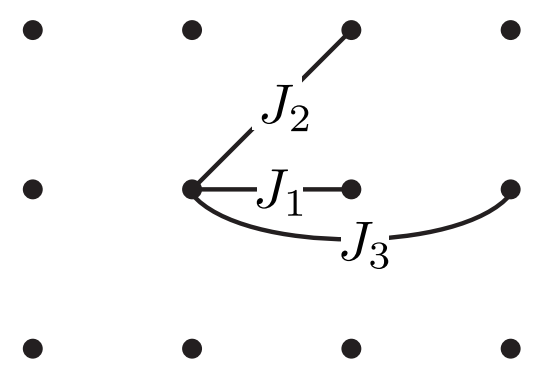

Fig. 8 The $J_{1}-J_{2}-J_{3}$ antiferromagnet. Spin $S$ spins are placed on each site of the square lattice, and they are coupled to all first, second, and third neighbors as shown. The Hamiltonian has the full space group symmetry of the square lattice, and there is only one spin per unit cell.

the group $S U(2)$ the complete set of $(2 S+1)$ states on site $i$ are represented as follows

$$
|S, m\rangle \equiv \frac{1}{\sqrt{(S+m) !(S-m) !}}\left(b_{i \uparrow}^{\dagger}\right)^{S+m}\left(b_{i \downarrow}^{\dagger}\right)^{S-m}|0\rangle,
$$

where $m=-S, \ldots S$ is the $z$ component of the spin ( $2 m$ is an integer). We have introduced two flavors of bosons on each site, created by the canonical operator $b_{i \alpha}^{\dagger}$, with $\alpha=\uparrow, \downarrow$, and $|0\rangle$ is the vacuum with no bosons. The total number of bosons, $n_{b}$ is the same for all the states; therefore

$$
b_{i \alpha}^{\dagger} b_{i}^{\alpha}=n_{b}
$$

with $n_{b}=2 S$ (we will henceforth assume an implied summation over repeated upper and lower indices). It is not difficult to see that the above representation of the states is completely equivalent to the following operator identity between the spin and boson operators

$$
S_{i a}=\frac{1}{2} b_{i \alpha}^{\dagger} \sigma_{\beta}^{a \alpha} b_{i}^{\beta},
$$

where $a=x, y, z$ and the $\sigma^{a}$ are the usual $2 \times 2$ Pauli matrices. The spin-states on two sites $i, j$ can combine to form a singlet in a unique manner - the wavefunction of the singlet state is particularly simple in the boson formulation:

$$
\left(\varepsilon^{\alpha \beta} b_{i \alpha}^{\dagger} b_{j \beta}^{\dagger}\right)^{2 S}|0\rangle \text {. }
$$

Finally we note that, using the constraint (17), the following Fierz-type identity can be established

$$
\left(\varepsilon^{\alpha \beta} b_{i \alpha}^{\dagger} b_{j \beta}^{\dagger}\right)\left(\varepsilon_{\gamma \delta} b_{i}^{\gamma} b_{j}^{\delta}\right)=-2 \boldsymbol{S}_{i} \cdot \boldsymbol{S}_{j}+n_{b}^{2} / 2+\delta_{i j} n_{b}
$$

where $\varepsilon$ is the totally antisymmetric $2 \times 2$ tensor

$$
\varepsilon=\left(\begin{array}{cc}
0 & 1 \\
-1 & 0
\end{array}\right)
$$


This implies that $\mathcal{H}$ can be rewritten in the form (apart from an additive constant)

$$
\mathcal{H}=-\frac{1}{2} \sum_{\langle i j\rangle} J_{i j}\left(\varepsilon^{\alpha \beta} b_{i \alpha}^{\dagger} b_{j \beta}^{\dagger}\right)\left(\varepsilon_{\gamma \delta} b_{i}^{\gamma} b_{j}^{\delta}\right) .
$$

This form makes it clear that $\mathcal{H}$ counts the number of singlet bonds.

We have so far defined a one-parameter $\left(n_{b}\right)$ family of models $\mathcal{H}$ for a fixed realization of the $J_{i j}$. Increasing $n_{b}$ makes the system more classical and a large $n_{b}$ expansion is therefore not suitable for studying the quantum-disordered phase. For this reason we introduce a second parameter - the flavor index $\alpha$ on the bosons is allowed to run from $1 \ldots 2 N$ with $N$ an arbitrary integer. This therefore allows the bosons to transform under $S U(2 N)$ rotations. However the $S U(2 N)$ symmetry turns out to be too large. We want to impose the additional restriction that the spins on a pair of sites be able to combine to form a singlet state, thus generalizing the valence-bond structure of $S U(2)$ - this valence-bond formation is clearly a crucial feature determining the structure of the quantum disordered phase. It is well-known that this is impossible for $S U(2 N)$ for $N>1$ - there is no generalization of the second-rank, antisymmetric, invariant tensor $\varepsilon$ to general $S U(2 N)$.

The proper generalization turns out to be to the group $S p(N)$ [54]. This group is defined by the set of $2 N \times 2 N$ unitary matrices $U$ such that

$$
U^{T} \mathcal{J} U=\mathcal{J}
$$

where

$$
\mathcal{J}_{\alpha \beta}=\mathcal{J}^{\alpha \beta}=\left(\begin{array}{cccc}
1 & & & \\
-1 & & & \\
& & 1 & \\
& -1 & & \\
& & \ddots & \\
& & & \ddots
\end{array}\right)
$$

is the generalization of the $\varepsilon$ tensor to $N>1$; it has $N$ copies of $\varepsilon$ along the diagonal. It is clear that $S p(N) \subset S U(2 N)$ for $N>1$, while $S p(1) \cong S U(2)$. The $b_{i}^{\alpha}$ bosons transform as the fundamental representation of $S p(N)$; the "spins" on the lattice therefore belong to the symmetric product of $n_{b}$ fundamentals, which is also an irreducible representation. Valence bonds

$$
\mathcal{J}^{\alpha \beta} b_{i \alpha}^{\dagger} b_{j \alpha}^{\dagger}
$$

can be formed between any two sites; this operator is a singlet under $S p(N)$ because of 23. The form 22, of $\mathcal{H}$ has a natural generalization to general $S p(N)$ :

$$
\mathcal{H}=-\sum_{i>j} \frac{J_{i j}}{2 N}\left(\mathcal{J}^{\alpha \beta} b_{i \alpha}^{\dagger} b_{j, \beta}^{\dagger}\right)\left(\mathcal{J}_{\gamma \delta} b_{i}^{\gamma} b_{j}^{\delta}\right)
$$




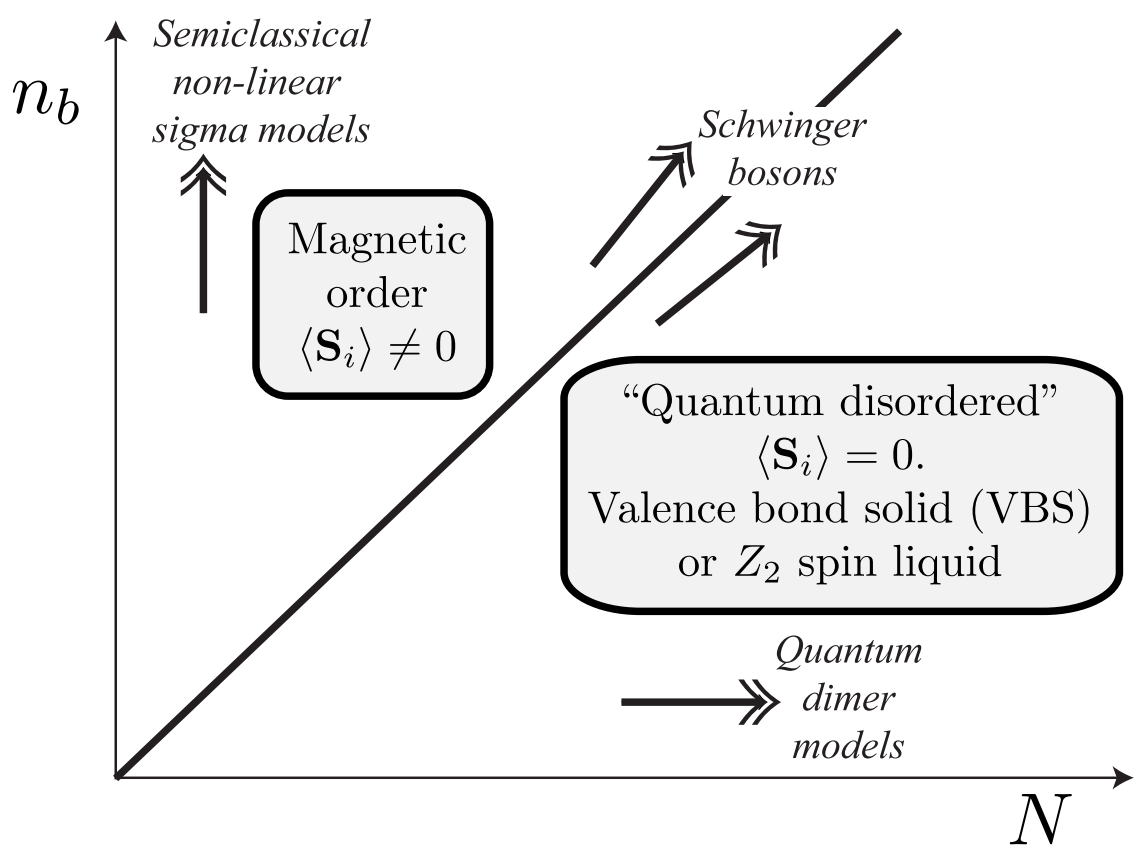

Fig. 9 Phase diagram of the 2D $S p(N)$ antiferromagnet $\mathcal{H}$ as a function of the "spin" $n_{b}$; from Refs. [54 59, 62] The "quantum disordered" region preserves $\operatorname{Sp}(N)$ spin rotation invariance, and there is no magnetic long-range order; however, the ground states here have new types of emergent order (VBS or $Z_{2}$ topological order), which are described in the text. On the square lattice, the $Z_{2}$ spin liquid phases also break a global lattice rotational symmetry, and so they have 'Ising-nematic' order; the $Z_{2}$ spin liquids on the triangular and kagome lattices do not break any lattice symmetry.

where the indices $\alpha, \beta, \gamma, \delta$ now run over $1 \ldots 2 N$. We recall also that the constraint (17) must be imposed on every site of the lattice.

We now have a two-parameter $\left(n_{b}, N\right)$ family of models $\mathcal{H}$ for a fixed realization of the $J_{i j}$. It is very instructive to consider the phase diagram of $\mathcal{H}$ as a function of these two parameters (Fig. 9p.

The limit of large $n_{b}$, with $N$ fixed leads to the semi-classical theory. For the special case of $S U(2)$ antiferromagnets with a two-sublattice collinear Néel ground state, the semiclassical fluctuations are described by the $O(3)$ non-linear sigma model. For other models [59, 63-68], the structure of the non-linear sigma models is rather more complicated and will not be considered here.

A second limit in which the problem simplifies is $N$ large at fixed $n_{b}$ [59, 69]. It can be shown that in this limit the ground state is quantum disordered. Further, the low-energy dynamics of $\mathcal{H}$ is described by an effective quantum-dimer model [59.70], with each dimer configuration representing a particular pairing of the sites into valence-bonds. There have been extensive studies of such quantum dimer models which we will not review here. All the quantum dimer model stud- 
ies in the "quantum disordered" region of Fig. 9 have yielded phases which were obtained earlier [54] by the methods to be described below.

The most interesting solvable limit is obtained by fixing the ratio of $n_{b}$ and $N$

$$
\kappa=\frac{n_{b}}{N}
$$

and subsequently taking the limit of large $N$ [58]; this limit will be studied in this section in considerable detail. The implementation of $\mathcal{H}$ in terms of bosonic operators also turns out to be naturally suited for studying this limit. The parameter $\kappa$ is arbitrary; tuning $\kappa$ modifies the slope of the line in Fig. 9 along which the large $N$ limit is taken. From the previous limits discussed above, one might expect that the ground state of $\mathcal{H}$ has magnetic long range order (LRO) for large $\kappa$ and is quantumdisordered for small $\kappa$. We will indeed find below that for any set of $J_{i j}$ there is a critical value of $\kappa=\kappa_{c}$ which separates the magnetically ordered and the quantum disordered phase.

The transition at $\kappa=\kappa_{c}$ is second-order at $N=\infty$, and is a powerful feature of the present large- $N$ limit. In the vicinity of the phase transition, we expect the physics to be controlled by long-wavelength, low-energy spin fluctuations; the large$N$ method offers an unbiased guide in identifying the proper low-energy degress of freedom and determines the effective action controlling them. Having obtained a long-wavelength continuum theory near the transition, one might hope to analyze the continuum theory independently of the large- $N$ approximation and obtain results that are more generally valid.

We will discuss the structure of the $N=\infty$ mean-field theory, with $n_{b}=\kappa N$ in Section 2.2.1 The long-wavelength effective actions will be derived and used to describe general properties of the phases and the phase transitions in Section 2.2.2

\subsubsection{Mean-field theory}

We begin by analyzing $\mathcal{H}$ at $N=\infty$ with $n_{b}=\kappa N$. As noted above, this limit is most conveniently taken using the bosonic operators. We may represent the partition function of $\mathcal{H}$ by

$$
Z=\int \mathcal{D} Q \mathcal{D} b \mathcal{D} \lambda \exp \left(-\int_{0}^{\beta} \mathcal{L} d \tau\right)
$$

where

$$
\begin{aligned}
\mathcal{L}= & \sum_{i}\left[b_{i \alpha}^{\dagger}\left(\frac{d}{d \tau}+i \lambda_{i}\right) b_{i}^{\alpha}-i \lambda_{i} n_{b}\right] \\
& +\sum_{\langle i, j\rangle}\left[N \frac{J_{i j}\left|Q_{i, j}\right|^{2}}{2}-\frac{J_{i j} Q_{i, j}^{*}}{2} \mathcal{J}_{\alpha \beta} b_{i}^{\alpha} b_{j}^{\beta}+H . c .\right] .
\end{aligned}
$$


Here the $\lambda_{i}$ fix the boson number of $n_{b}$ at each site; $\tau$-dependence of all fields is implicit. The complex field $Q$ was introduced by a Hubbard-Stratonovich decoupling of $\mathcal{H}$ : performing the functional integral over $Q$ reproduces the exchange coupling in Eq. 26. An important feature of the lagrangian $\mathcal{L}$ is its $U(1)$ gauge invariance under which

$$
\begin{aligned}
b_{i \alpha}^{\dagger} & \rightarrow b_{i \alpha}^{\dagger}(i) \exp \left(i \rho_{i}(\tau)\right) \\
Q_{i, j} & \rightarrow Q_{i, j} \exp \left(-i \rho_{i}(\tau)-i \rho_{j}(\tau)\right) \\
\lambda_{i} & \rightarrow \lambda_{i}+\frac{\partial \rho_{i}}{\partial \tau}(\tau) .
\end{aligned}
$$

The functional integral over $\mathcal{L}$ faithfully represents the partition function apart from an overall factor associated with this gauge redundancy.

The $1 / N$ expansion of the free energy can be obtained by integrating out of $\mathcal{L}$ the $2 N$-component $b, \bar{b}$ fields to leave an effective action for $Q, \lambda$ having coefficient $N$ (because $n_{b} \propto N$ ). Thus the $N \rightarrow \infty$ limit is given by minimizing the effective action with respect to "mean-field" values of $Q=\bar{Q}, i \lambda=\bar{\lambda}$ (we are ignoring here the possibility of magnetic LRO which requires an additional condensate $x^{\alpha}=\left\langle b^{\alpha}\right\rangle$ - this has been discussed elsewhere [54, 62]). This is in turn equivalent to solving the mean-field Hamiltonian

$$
\begin{aligned}
\mathcal{H}_{M F}= & \sum_{\langle i, j\rangle}\left(N \frac{J_{i j}\left|\bar{Q}_{i j}\right|^{2}}{2}-\frac{J_{i j} \bar{Q}_{i, j}^{*}}{2} \mathcal{J}_{\alpha \beta} b_{i}^{\alpha} b_{j}^{\beta}+H . c .\right) \\
& +\sum_{i} \bar{\lambda}_{i}\left(b_{i \alpha}^{\dagger} b_{i}^{\alpha}-n_{b}\right) .
\end{aligned}
$$

This Hamiltonian is quadratic in the boson operators and all its eigenvalues can be determined by a Bogoliubov transformation. This leads in general to an expression of the form

$$
\mathcal{H}_{M F}=E_{M F}[\bar{Q}, \bar{\lambda}]+\sum_{\mu} \omega_{\mu}[\bar{Q}, \bar{\lambda}] \gamma_{\mu \alpha}^{\dagger} \gamma_{\mu}^{\alpha}
$$

The index $\mu$ extends over $1 \ldots$ number of sites in the system, $E_{M F}$ is the ground state energy and is a functional of $\bar{Q}, \bar{\lambda}, \omega_{\mu}$ is the eigenspectrum of excitation energies which is a also a function of $\bar{Q}, \bar{\lambda}$, and the $\gamma_{\mu}^{\alpha}$ represent the bosonic eigenoperators. The excitation spectrum thus consists of non-interacting spinor bosons. The ground state is determined by minimizing $E_{M F}$ with respect to the $\bar{Q}_{i j}$ subject to the constraints

$$
\frac{\partial E_{M F}}{\partial \bar{\lambda}_{i}}=0 .
$$

The saddle-point value of the $\bar{Q}$ satisfies

$$
\bar{Q}_{i j}=\left\langle\mathcal{J}_{\alpha \beta} b_{i}^{\alpha} b_{j}^{\beta} \cdot\right\rangle
$$


Note that $\bar{Q}_{i j}=-\bar{Q}_{j i}$ indicating that $\bar{Q}_{i j}$ is a directed field - an orientation has to be chosen on every link.

We now describe the ground state configurations of the $\bar{Q}, \bar{\lambda}$ fields and the nature of the bosonic eigenspectrum for the $J_{1}-J_{2}-J_{3}$ model. We examined the values of the energy $E_{M F}$ for $\bar{Q}_{i j}$ configurations which had a translational symmetry with two sites per unit cell. For all parameter values configurations with a single site per unit cell were always found to be the global minima. We will therefore restrict our attention to such configurations. The $\bar{\lambda}_{i}$ field is therefore independent of $i$, while there are six independent values of $\bar{Q}_{i j}$ :

$$
\begin{aligned}
\bar{Q}_{i, i+\hat{x}} & \equiv Q_{1, x} \\
\bar{Q}_{i, i+\hat{y}} & \equiv Q_{1, y} \\
\bar{Q}_{i, i+\hat{y}+\hat{x}} & \equiv Q_{2, y+x} \\
\bar{Q}_{i, i+\hat{y}-\hat{x}} & \equiv Q_{2, y-x} \\
\bar{Q}_{i, i+2 \hat{x}} & \equiv Q_{3, x} \\
\bar{Q}_{i, i+2 \hat{y}} & \equiv Q_{3, y}
\end{aligned}
$$

For this choice, the bosonic eigenstates are also eigenstates of momentum with momenta $k$ extending over the entire first Brillouin zone. The bosonic eigenenergies are given by

$$
\begin{aligned}
\omega_{\boldsymbol{k}}= & \left(\bar{\lambda}^{2}-\left|A_{\boldsymbol{k}}\right|^{2}\right)^{1 / 2} \\
A_{\boldsymbol{k}}= & J_{1}\left(Q_{1, x} \sin k_{x}+Q_{1, y} \sin k_{y}\right) \\
& +J_{2}\left(Q_{2, y+x} \sin \left(k_{y}+k_{x}\right)+Q_{2, y-x} \sin \left(k_{y}-k_{x}\right)\right) \\
& +J_{3}\left(Q_{3, x} \sin \left(2 k_{x}\right)+Q_{3, y} \sin \left(2 k_{y}\right)\right) .
\end{aligned}
$$

We have numerically examined the global minima of $E_{M F}$ as a function of the three parameters $J_{2} / J_{1}, J_{3} / J_{1}$, and $N / n_{b}$ [54 62]. The values of the $\bar{Q}_{i j}$ at any point in the phase diagram can then be used to classify the distinct classes of states. The results are summarized in Figs. 10 and 11 which show two sections of the three-dimensional phase diagram. All of the phases are labeled by the wavevector at which the spin structure factor has a maximum. This maximum is a delta function for the phases with magnetic LRO, while it is simply a smooth function of $\boldsymbol{k}$ for the quantum disordered phases (denoted by SRO in Figs. 10 and 11). The location of this maximum will simply be twice the wavevector at which $\omega_{k}$ has a mimimum: this is because the structure factor involves the product of two bosonic correlation functions, each of which consists of a propagator with energy denominator $\omega_{\boldsymbol{k}}$.

Each of the phases described below has magnetic LRO for large $n_{b} / N$ and is quantum disordered for small $n_{b} / N$. The mean-field result for the structure of all of the quantum disordered phases is also quite simple: they are featureless spin fluids with free spin-1/2 bosonic excitations ("spinons") with energy dispersion $\omega_{\boldsymbol{k}}$ which is gapped over the entire Brillouin zone. Some of the quantum disordered phases break the lattice rotation symmetry (leading to 'Ising-nematic' order) even 


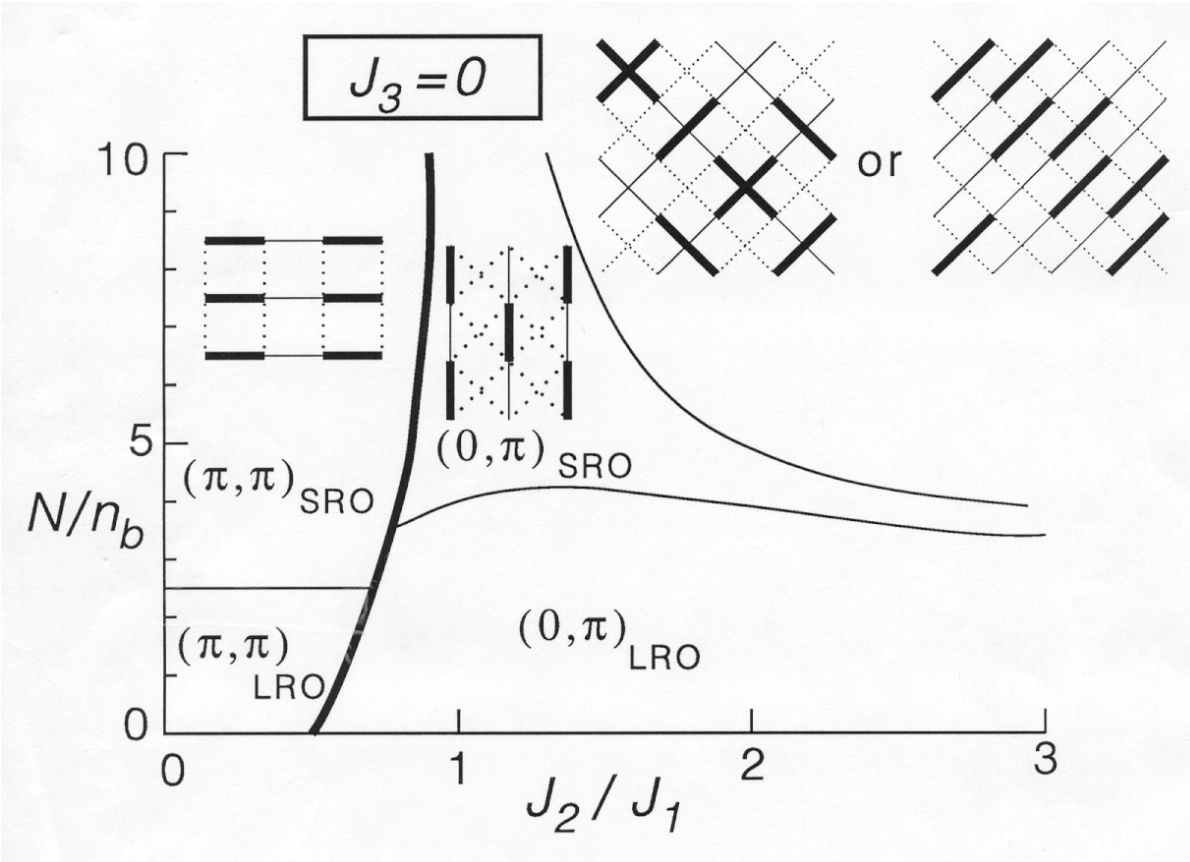

Fig. 10 Ground states of the $J_{1}-J_{2}-J_{3}$ model for $J_{3}=0$ as a function of $J_{2} / J_{1}$ and $N / n_{b}\left(n_{b}=\right.$ $2 S$ for $S U(2)$ ). Thick (thin) lines denote first (second) order transitions at $N=\infty$. Phases are identified by the wavevectors at which they have magnetic long-range-order (LRO) or short-rangeorder (SRO); the SRO phases are "quantum disordered" as in Fig. 9 The links with $Q_{p} \neq 0$ in each SRO phase are shown. The large $N / n_{b}$, large $J_{2} / J_{1}$ phase has the two sublattices decoupled at $N=\infty$. All LRO phases above have two-sublattice collinear Néel order. All the SRO phases above have valence bond solid (VBS) order at finite $N$ for odd $n_{b}$; this is illustrated by the thick, thin and dotted lines.

at $N=\infty$ - these will be described below. The mininum energy spinons lie at a wavevector $\boldsymbol{k}_{0}$ and $\omega_{\boldsymbol{k}_{0}}$ decreases as $n_{b} / N$. The onset of magnetic LRO occurs at the value of $n_{b} / N$ at which the gap first vanishes: $\omega_{\boldsymbol{k}_{0}}=0$. At still larger values of $n_{b} / N$, we get macroscopic bose condensation of the $b$ quanta at the wavevector $\boldsymbol{k}_{0}$, leading to magnetic LRO at the wavevector $2 \boldsymbol{k}_{0}$.

We now turn to a description of the various phases obtained. They can be broadly classified into two types:

\section{Commensurate collinear phases}

In these states the wavevector $\boldsymbol{k}_{0}$ remains pinned at a commensurate point in the Brillouin zone, which is independent of the values of $J_{2} / J_{1}, J_{3} / J_{1}$ and $n_{b} / N$. In the LRO phase, the spin condensates on the sites are either parallel or anti-parallel to each other, which we identify as collinear ordering. This implies that the LRO 


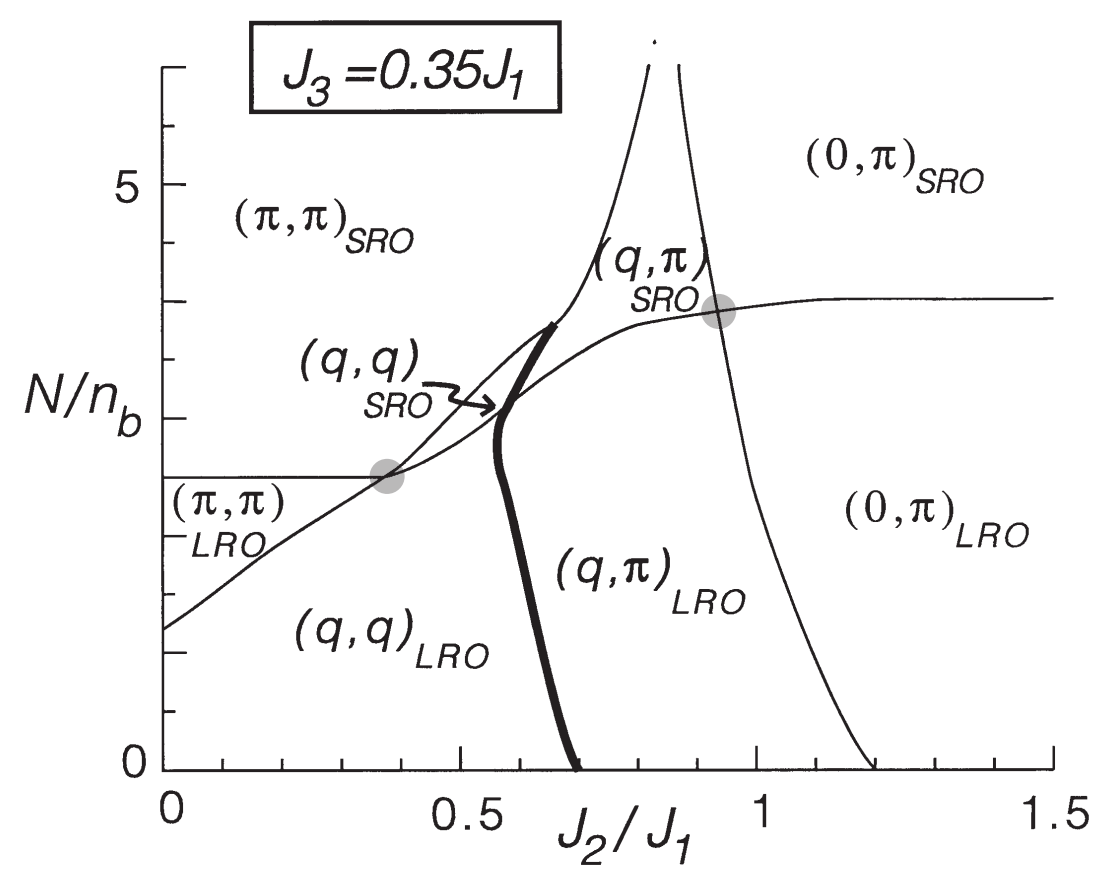

Fig. 11 As in Fig. 10 but for $J_{3} / J_{1}=0.35$. The $(0, \pi)_{S R O}$ and $(\pi, \pi)_{S R O}$ phases have VBS order as illustrated in Fig. 10 The $(q, q)_{S R O}$ and $(q, \pi)_{S R O}$ phases are $Z_{2}$ spin liquids: they have topological order, and a topological 4-fold degeneracy of the ground state on the torus. The $Z_{2}$ spin liquids here also have Ising-nematic order, i.e., they break the $90^{\circ}$ rotation symmetry of the square lattice, which leads to an additional 2-fold degeneracy. The $(q, q)_{L R O}$ and $(q, \pi)_{L R O}$ have magnetic long-range order in the form of an incommensurate spiral. The 2 shaded circles indicate regions which map onto the generalized phase diagram in Fig. 12

phase remains invariant under rotations about the condensate axis and the rotation symmetry is not completely broken.

Three distinct realizations of such states were found

a. $(\pi, \pi)$

This is the usual two-sublattice Néel state of the unfrustrated square lattice and its quantum-disordered partner. These states have

$$
Q_{1, x}=Q_{1, y} \neq 0, Q_{2, y+x}=Q_{2, y-x}=Q_{3, x}=Q_{3, y}=0 .
$$

From (36), the minimum spinon excitation occurs at $\boldsymbol{k}_{0}= \pm(\pi / 2, \pi / 2)$. The SRO states have no broken symmetry at $N=\infty$. The boundary between the LRO and SRO phases occurs at $N / n_{b}<2.5$, independent of $J_{2} / J_{1}$ (Fig. 10). This last feature 
is surely an artifact of the large $N$ limit. Finite $N$ fluctuations should be stronger as $J_{2} / J_{1}$ increases, causing the boundary to bend a little downwards to the right.

b. $(\pi, 0)$ or $(0, \pi)$

The $(0, \pi)$ states have

$$
Q_{1, x}=0, Q_{1, y} \neq 0, Q_{2, y+x}=Q_{2, y-x} \neq 0, \text { and } Q_{3, x}=Q_{3, y}=0
$$

and minimum energy spinons at $\boldsymbol{k}_{0}= \pm(0, \pi / 2)$. The degenerate $(\pi, 0)$ state is obtained with the mapping $x \leftrightarrow y$. The SRO state has a two-field degeneracy due to the broken $x \leftrightarrow y$ lattice symmetry: the order associated with this symmetry is referred to as 'Ising-nematic' order. We can use the $Q$ variables here to define an Ising nematic order parameter

$$
\mathcal{I}=\left|Q_{1 x}\right|^{2}-\left|Q_{1 y}\right|^{2} .
$$

This is a gauge-invariant quantity, and the square lattice symmetry of the Hamiltonian implies that $\langle\mathcal{I}\rangle=0$ unless the symmetry is spontaneously broken. The sign of $\langle\mathcal{I}\rangle$ chooses between the $(\pi, 0)$ and $(0, \pi)$ states. The LRO state again has twosublattice collinear Néel order, but the assignment of the sublattices is different from the $(\pi, \pi)$ state. The spins are parallel along the $x$-axis, but anti-parallel along the $y$-axis.

An interesting feature of the LRO state here is the occurrence of "order-fromdisorder" [71]. The classical limit $\left(n_{b} / N=\infty\right)$ of this model has an accidental degeneracy for $J_{2} / J_{1}>1 / 2$ : the ground state has independent collinear Néel order on each of the $A$ and $B$ sublattices, with the energy independent of the angle between the spins on the two sublattices. Quantum fluctuations are included self-consistently in the $N=\infty, n_{b} / N$ finite, mean-field theory, and lead to an alignment of the spins on the sublattices and LRO at $(0, \pi)$. The orientation of the ground state has thus been selected by the quantum fluctuations.

The $(0, \pi)$ states are separated from the $(\pi, \pi)$ states by a first-order transition. In particular, the spin stiffnesses of both states remain finite at the boundary between them. This should be distinguished from the classical limit in which the stiffness of both states vanish at their boundary $J_{2}=J_{1} / 2$; the finite spin stiffnesses are thus another manifestation of order-from-disorder. At a point well away from the singular point $J_{2}=J_{1} / 2, n_{b} / N=\infty$ in Fig. 10, the stiffness of both states is of order $N\left(n_{b} / N\right)^{2}$ for $N=\infty$ and large $n_{b} / N$; near this singular point however the stiffness is of order $N\left(n_{b} / N\right)$ and is induced purely by quantum fluctuations. These results have also been obtained by a careful resummation of the semiclassical expansion [72] 73]. 


\section{c. "Decoupled"}

For $J_{2} / J_{1}$ and $N / n_{b}$ both large, we have a "decoupled" state (Fig. 10 with

$$
Q_{2, y+x}=Q_{2, y-x} \neq 0 \text { and } Q_{1}=Q_{3}=0 .
$$

In this case $Q_{p}$ is non-zero only between sites on the same sublattice. The two sublattices have Néel type SRO which will be coupled by finite $N$ fluctuations. The $N=\infty$ state does not break any lattice symmetry. This state has no LRO partner.

\section{Incommensurate phases}

In these phases the wavevector $\boldsymbol{k}_{0}$ and the location of the maximum in the structure factor move continuously with the parameters. The spin-condensate rotates with a period which is not commensurate with the underlying lattice spacing. Further the spin condensate is coplanar: the spins rotate within a given plane in spin space and are not collinear. There is no spin rotation axis about which the LRO state remains invariant.

Further, no states in which the spin condensate was fully three dimensional ("double-spiral" or chiral states) were found; these would be associated with complex values of $Q_{p}$. All the saddle points possesed a gauge in which all the $Q_{p}$ were real. Time-reversal symmetry was therefore always preserved in all the SRO phases of Figs. 10 and 11 .

The incommensurate phases occur only in models with a finite $J_{3}$ (Fig. 11, at least at $N=\infty$. There were two realizations:

\section{d. $(\pi, q)$ or $(q, \pi)$}

Here $q$ denotes a wavevector which varies continuously between 0 and $\pi$ as the parameters are changed. The $(q, \pi)$ state has

$$
Q_{1, x} \neq Q_{1, y} \neq 0, Q_{2, x+y}=Q_{2, y-x} \neq 0, Q_{3, x} \neq 0 \text { and } Q_{3, y}=0 ;
$$

the degenerate $(\pi, q)$ helix is obtained by the mapping $x \leftrightarrow y$. The SRO state has a two-fold degeneracy due to the broken $x \leftrightarrow y$ lattice symmetry, and so this state has Ising-nematic order. The order parameter in Eq. (39) continues to measure this broken symmetry.

\section{e. $(q, q)$ or $(q,-q)$}

The $(q, q)$ state has

$$
Q_{1, x}=Q_{1, y} \neq 0, Q_{2, x+y} \neq 0, Q_{2, y-x}=0, Q_{3, x}=Q_{3, y} \neq 0 ;
$$


this is degenerate with the $(q,-q)$ phase. The SRO state therefore has a two-fold degeneracy due to a broken lattice reflection symmetry, and so it also has Ising nematic order. However, the Ising symmetry now corresponds to reflections about the principle square axes, and the analog of Eq. 39 , is now

$$
\mathcal{I}=\left|Q_{2, x+y}\right|^{2}-\left|Q_{2, y-x}\right|^{2} .
$$

As we noted above, the broken discrete symmetries in states with SRO at $(0, \pi)$ and $(q, \pi)$ are identical: both are two-fold degenerate due to a breaking of the $x \leftrightarrow y$ symmetry. The states are only distinguished by a non-zero value of $Q_{3}$ in the $(q, \pi)$ phase and the accompanying incommensurate correlations in the spin-spin correlation functions. However $Q_{3}$ is gauge-dependent and so somewhat unphysical as an order parameter. In the absence of any further fluctuation-driven lattice symmetry breaking, the transition between SRO at $(0, \pi)$ and $(q, \pi)$ is an example of a disorder line [74]; these are lines at which incommensurate correlations first turn on. However, we will see that quantum fluctuations clearly distinguish these two phases, which have confined and deconfined spinons respectively, and the associated topological order requires a phase transitions between them.

An interesting feature of Fig. 11 is that the commensurate states squeeze out the incommensurate phases as $N / n_{b}$ increases. We expect that this suppression of incommensurate order by quantum fluctuations is a general feature of frustrated antiferromagnets.

\subsubsection{Fluctuations - long wavelength effective actions}

We now extend the analysis of Section 2.2.1 beyond the mean-field theory and examine the consequences of corrections at finite $N$. The main question we hope to address are:

- The mean-field theory yielded an excitation spectrum consisting of free spin-1/2 bosonic spinons. We now want to understand the nature of the forces between these spinons and whether they can lead to confinement of half-integer spin excitations.

- Are there any collective excitations and does their dynamics modify in any way the nature of the mean field ground state ?

The structure of the fluctuations will clearly be determined by the low-energy excitations about the mean-field state. We have already identified one set of such excitations: spinons at momenta near mimima in their dispersion spectrum, close to the onset of the magnetic LRO phase whence the spinon gap vanishes. An additional set of low-lying spinless excitations can arise from the fluctuations of the $Q_{i j}$ and $\lambda_{i}$ fields about their mean-field values. The gauge-invariance (30) will act as a powerful restriction on the allowed terms in the effective action for these spinless fields. We anticipate that the only such low-lying excitations are associated with the $\lambda_{i}$ and the phases of the $Q_{i j}$. We therefore parametrize 


$$
Q_{i, i+\hat{e}_{p}}=\bar{Q}_{i, i+\hat{e}_{p}} \exp \left(-i \Theta_{p}\right)
$$

where the vector $\hat{e}_{p}$ connects the two sites of the lattice under consideration, $\bar{Q}$ is the mean-field value, and $\Theta_{p}$ is a real phase. The gauge invariance 30 implies that the effective action for the $\Theta_{p}$ must be invariant under

$$
\Theta_{p} \rightarrow \Theta_{p}+\rho_{i}+\rho_{i+\hat{e}_{p}} .
$$

Upon performing a Fourier transform, with the link variables $\Theta_{p}$ placed on the center of the links, the gauge invariance takes the form

$$
\Theta_{p}(\boldsymbol{k}) \rightarrow \Theta_{p}(\boldsymbol{k})+2 \rho(\boldsymbol{k}) \cos \left(k_{p} / 2\right),
$$

where $k_{p}=\boldsymbol{k} \cdot \hat{e}_{p}$. This invariance implies that the effective action for the $\Theta_{p}$, after integrating out the $b$ quanta, can only be a function of the following gauge-invariant combinations:

$$
I_{p q}=2 \cos \left(k_{q} / 2\right) \Theta_{p}(\boldsymbol{k})-2 \cos \left(k_{p} / 2\right) \Theta_{q}(\boldsymbol{k}) .
$$

We now wish to take the continuum limit at points in the Brillouin zone where the action involves only gradients of the $\Theta_{p}$ fields and thus has the possibility of gapless excitations. This involves expanding about points in the Brillouin zone where

$$
\cos \left(k_{p} / 2\right)=0 \text { for the largest numbers of } \hat{e}_{p} .
$$

We now apply this general principle to the $J_{1^{-}} J_{2^{-}} J_{3}$ model.

\section{Commensurate collinear phases}

We begin by examining the $(\pi, \pi)$-SRO phase. As noted in 37p, this phase has the mean field values $Q_{1, x}=Q_{1, y} \neq 0$, and all other $\bar{Q}_{i j}$ zero. Thus we need only examine the condition (48) with $\hat{e}_{p}=\hat{e}_{x}, \hat{e}_{y}$. This uniquely identifies the point $\boldsymbol{k}=\boldsymbol{G}=(\pi, \pi)$ in the Brillouin zone. We therefore parametrize

$$
\Theta_{x}(\boldsymbol{r})=e^{i \boldsymbol{G} \cdot \boldsymbol{r}} A_{x}(\boldsymbol{r})
$$

and similarly for $\Theta_{y}$; it can be verified that both $\Theta$ and $A_{x}$ are real in the above equation. We will also be examining invariances of the theory under gauge transformations near $G$ : so we write

$$
\rho(\boldsymbol{r})=e^{i \boldsymbol{G} \cdot \boldsymbol{r}} \zeta(\boldsymbol{r}) .
$$

It is now straightforward to verify that the gauge transformations (46) are equivalent to

$$
A_{x} \rightarrow A_{x}+\partial_{x} \zeta
$$


and similarly for $A_{y}$. We will also need in the continuum limit the component of $\lambda$ near the wavevector $\boldsymbol{G}$. We therefore write

$$
i \lambda_{i}=\bar{\lambda}+i e^{i \boldsymbol{G} \cdot \boldsymbol{r}} A_{\tau}\left(\boldsymbol{r}_{i}\right) .
$$

Under gauge transformations we have

$$
A_{\tau} \rightarrow A_{\tau}+\partial_{\tau} \zeta
$$

Thus $A_{x}, A_{y}, A_{\tau}$ transform as components of a continuum $U(1)$ vector gauge field.

We will also need the properties of the boson operators under the gauge transformation $\zeta$. From (30) and (50) we see that the bosons on the two sublattices $(A, B)$ carry opposite charges \pm 1 :

$$
\begin{aligned}
& b_{A} \rightarrow b_{A} e^{i \zeta} \\
& b_{B} \rightarrow b_{B} e^{-i \zeta} .
\end{aligned}
$$

Finally, we note that the bosonic eigenspectrum has a minimum near $\boldsymbol{k}=\boldsymbol{k}_{0}=$ $(\pi / 2, \pi / 2)$; we therefore parametrize

$$
\begin{aligned}
b_{A i}^{\alpha} & =\psi_{1}^{\alpha}\left(\boldsymbol{r}_{i}\right) e^{i \boldsymbol{k}_{0} \cdot \boldsymbol{r}_{i}} \\
b_{B i}^{\alpha} & =-i \mathcal{J}^{\alpha \beta} \psi_{2 \beta}\left(\boldsymbol{r}_{i}\right) e^{i \boldsymbol{k}_{0} \cdot \boldsymbol{r}_{i}} .
\end{aligned}
$$

We insert the continuum parameterizations (49), (52) and (55) into the functional integral 29 , perform a gradient expansion, and transform the Lagrangian $\mathcal{L}$ into

$$
\begin{gathered}
\mathcal{L}=\int \frac{d^{2} r}{a^{2}}\left[\psi_{1 \alpha}^{*}\left(\frac{d}{d \tau}+i A_{\tau}\right) \psi_{1}^{\alpha}+\psi_{2}^{\alpha *}\left(\frac{d}{d \tau}-i A_{\tau}\right) \psi_{2 \alpha}\right. \\
+\bar{\lambda}\left(\left|\psi_{1}^{\alpha}\right|^{2}+\left|\psi_{2 \alpha}\right|^{2}\right)-4 J_{1} \bar{Q}_{1}\left(\psi_{1}^{\alpha} \psi_{2 \alpha}+\psi_{1 \alpha}^{*} \psi_{2}^{\alpha *}\right) \\
+J_{1} \bar{Q}_{1} a^{2}\left[(\boldsymbol{\nabla}+i \boldsymbol{A}) \psi_{1}^{\alpha}(\boldsymbol{\nabla}-i \boldsymbol{A}) \psi_{2 \alpha}\right. \\
\left.\left.+(\boldsymbol{\nabla}-i \boldsymbol{A}) \psi_{1 \alpha}^{*}(\boldsymbol{\nabla}+i \boldsymbol{A}) \psi_{2}^{\alpha *}\right]\right]
\end{gathered}
$$

We now introduce the fields

$$
\begin{aligned}
z^{\alpha} & =\left(\psi_{1}^{\alpha}+\psi_{2}^{\alpha *}\right) / \sqrt{2} \\
\pi^{\alpha} & =\left(\psi_{1}^{\alpha}-\psi_{2}^{\alpha *}\right) / \sqrt{2} .
\end{aligned}
$$

Following the definitions of the underlying spin operators, it is not difficult to show that the Néel order parameter $\varphi_{a}$ (which is proportional to $\boldsymbol{n}$ in (2)) is related to the $z_{\alpha}$ by

$$
\varphi_{a}=z_{\alpha}^{*} \sigma_{\beta}^{a \alpha} z^{\beta} .
$$

From Eq. 56 , it is clear that the the $\pi$ fields turn out to have mass $\bar{\lambda}+4 J_{1} \bar{Q}_{1}$, while the $z$ fields have a mass $\bar{\lambda}-4 J_{1} \bar{Q}_{1}$ which vanishes at the transition to the LRO 
phase. The $\pi$ fields can therefore be safely integrated out, and $\mathcal{L}$ yields the following effective action, valid at distances much larger than the lattice spacing [60,61]:

$$
S_{\mathrm{eff}}=\int \frac{d^{2} r}{\sqrt{8} a} \int_{0}^{c \beta} d \tilde{\tau}\left\{\left|\left(\partial_{\mu}-i A_{\mu}\right) z^{\alpha}\right|^{2}+\frac{\Delta^{2}}{c^{2}}\left|z^{\alpha}\right|^{2}\right\} .
$$

Here $\mu$ extends over $x, y, \tau, c=\sqrt{8} J_{1} \bar{Q}_{1} a$ is the spin-wave velocity, $\Delta=\left(\bar{\lambda}^{2}-\right.$ $\left.16 J_{1}^{2} \bar{Q}_{1}^{2}\right)^{1 / 2}$ is the gap towards spinon excitations, and $A_{\tilde{\tau}}=A_{\tau} / c$. Thus, in its final form, the long-wavelength theory consists of a massive, spin-1/2, relativistic, boson $z^{\alpha}$ (spinon) coupled to a compact $U(1)$ gauge field. By 'compact' we mean that values $A_{\mu}$ and $A_{\mu}+2 \pi$ are identified with each other, and the gauge field lives on a circle: this is clearly required by Eq. (44).

At distances larger than $c / \Delta$, we may safely integrate out the massive $z$ quanta and obtain a a compact $U(1)$ gauge theory in $2+1$ dimensions. This theory was argued by Polyakov [75, 76] to be permanently in a confining phase, with the confinement driven by "monopole" tunnelling events. The compact $U(1)$ gauge force will therefore confine the $z^{\alpha}$ quanta in pairs. So the conclusion is that the $(\pi, \pi)_{S R O}$ does not possess $S=1 / 2$ spinon excitations, as was the case in the mean field theory. Instead, the lowest-lying excitations with non-zero spin will be triplons, similar to those in Section 2.1. A further important effect here, not present in the $U(1)$ gauge theories considered by Polyakov, is that the monopole tunnelling events carry Berry phases. The influence of these Berry phases has been described [60 61] and reviewed [39] elsewhere, and so will not be explained here. The result is that the condensation of monopoles with Berry phases leads to valence bond solid (VBS) order in the ground state. This order is associated with the breaking of the square lattice space group symmetry, as illustrated in Figs. 10 and 12 below. For the $(\pi, \pi)_{S R O}$ phase, this means that the singlet spin correlations have a structure similar to that in Fig. 4. In other words, the square lattice antiferromagnet spontaneously acquires a ground state with a symmetry similar to that of the paramagnetic phase of coupleddimer antiferromagnet. Because the VBS order is spontaneous, the ground state is 4-fold degenerate (associated with $90^{\circ}$ rotations about a lattice site), unlike the nondegenerate ground state of the dimerized antiferromagnet of Section 2.1. VBS states with a plaquette ordering pattern can also appear, but are not shown in the figures.

The quantum phase transition between the $(\pi, \pi)_{S R O}$ and $(\pi, \pi)_{L R O}$ phases has been the topic of extensive study. The proposal of Refs. [77, 78] is that monopoles are suppressed precisely at the quantum critical point, and so the continuum action in Eq. (58) constitutes a complete description of the critical degrees of freedom. It has to be supplemented by a quartic non-linearity $\left(\left|z^{\alpha}\right|^{2}\right)^{2}$, because such shortrange interactions are relevant perturbations at the critical point. A review of this deconfined criticality proposal is found elsewhere [37].

The properties of the $(0, \pi)$ phase are very similar to those of the $(\pi, \pi)$ phase considered above. It can be shown quite generally that any quantum disordered state which has appreciable commensurate, collinear spin correlations will have similar properties: confined spinons, a collective mode described by a compact $U(1)$ gauge field, and VBS order for odd $n_{b}$. 


\section{Incommensurate phases}

We now turn to a study of the incommensurate phases. It is not difficult to show that in this case it is not possible to satisfy the constraints (48) at any point in the Brillouin zone for all the non-zero $Q_{p}$. This implies that, unlike the commensurate phases, there is no gapless collective gauge mode in the gaussian fluctuations of the incommensurate SRO phases. This has the important implication that the meanfield theory is stable: the structure of the mean-field ground state, and its spinon excitations will survive fluctuation corrections. Thus we obtain a stable 'spin liquid' with bosonic $S=1 / 2$ spinon excitations. We will now show that these spinons carry a $Z_{2}$ gauge charge, and so this phase is referred to as a $Z_{2}$ spin liquid. The $Z_{2}$ gauge field also accounts for 'topological order' and a 4-fold ground state degeneracy on the torus.

The structure of the theory is simplest in the vicinity of a transition to a commensurate collinear phase: we now examine the effective action as one moves from the $(\pi, \pi)$-SRO phase into the $(q, q)$-SRO phase (Fig. 11, a very similar analysis can be performed at the boundary between the $(\pi, \pi)$-SRO and the $(\pi, q)$-SRO phases). This transition is characterized by a continuous turning on of non-zero values of $Q_{i, i+\hat{y}+\hat{x}}, Q_{i, i+2 \hat{x}}$ and $Q_{i, i+2 \hat{y}}$. It is easy to see from Eq. 30 ) that these fields transform as scalars of charge \pm 2 under the gauge transformation associated with $A_{\mu}$. Performing a gradient expansion upon the bosonic fields coupled to these scalars we find that the Lagrangian $\mathcal{L}$ of the $(\pi, \pi)$-SRO phase gets modified to

$$
\mathcal{L} \rightarrow \mathcal{L}+\int \frac{d^{2} r}{a}\left(\boldsymbol{\Lambda}_{A} \cdot\left(\mathcal{J}_{\alpha \beta} \psi_{1}^{\alpha} \nabla \psi_{1}^{\beta}\right)+\boldsymbol{\Lambda}_{B} \cdot\left(\mathcal{J}^{\alpha \beta} \psi_{2 \alpha} \nabla \psi_{2 \beta}\right)+\text { c.c. }\right)
$$

where $\boldsymbol{\Lambda}_{A, B}$ are two-component scalars $\equiv\left(J_{3} Q_{3, x}+J_{2} Q_{2, y+x}, J_{3} Q_{3, y}+J_{2}\right.$ $\left.Q_{2, y+x}\right)$ with the sites on the ends of the link variables on sublattices $A, B$. Finally, as before, we transform to the $z, \pi$ variables, integrate out the $\pi$ fluctuations and obtain [62]

$$
\begin{aligned}
S_{\mathrm{eff}}=\int \frac{d^{2} r}{\sqrt{8} a} \int_{0}^{c \beta} d \tilde{\tau}\left\{\left|\left(\partial_{\mu}-i A_{\mu}\right) z^{\alpha}\right|^{2}+s_{z}\left|z^{\alpha}\right|^{2}+\boldsymbol{\Lambda} \cdot\left(\mathcal{J}_{\alpha \beta} z^{\alpha} \nabla z^{\beta}\right)+\right.\text { c.c. } \\
\left.\quad+K_{\Lambda}\left|\left(\partial_{\mu}+2 i A_{\mu}\right) \boldsymbol{\Lambda}\right|^{2}+s_{\Lambda} \boldsymbol{\Lambda}^{2}+\text { terms quartic in } z^{\alpha}, \boldsymbol{\Lambda}\right\} .
\end{aligned}
$$

Here $s_{z}=\Delta^{2} / c^{2}, \boldsymbol{\Lambda}=\left(\boldsymbol{\Lambda}_{A}+\boldsymbol{\Lambda}_{B}^{*}\right) /\left(2 J_{1} \bar{Q}_{1} a\right)$ is a complex scalar of charge -2 , and $K_{\Lambda}$ is a stiffness. We have explicitly written the quadratic terms in the effective action for the $\boldsymbol{\Lambda}$ : these are generated by short wavelength fluctuations of the $b^{\alpha}$ quanta. We have omitted quartic and higher order terms which are needed to stabilize the theory when the 'masses' $s_{z}$ or $s_{\Lambda}$ are negative, and are also important near the quantum phase transitions. This effective action is also the simplest theory that can be written down which couples a spin-1/2, charge 1 , boson $z^{\alpha}$, a compact $U(1)$ gauge field $A_{\mu}$, and a two spatial component, charge -2 , spinless boson $\Lambda$. 


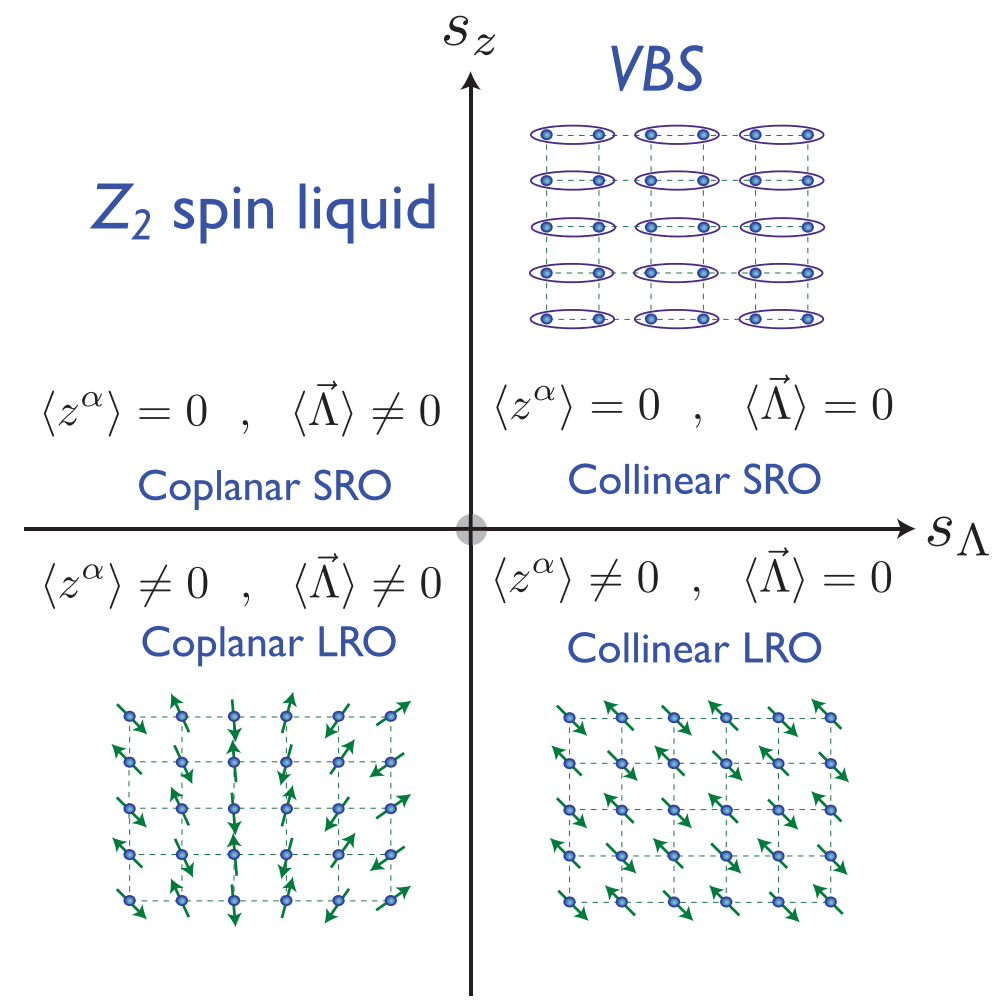

Fig. 12 Phase diagram of the theory $S_{\text {eff }}$ in Eq. 60 for the bosonic spinons $z_{\alpha}$ and the charge -2 spinless boson $\boldsymbol{\Lambda}$. Fig. 10 contains examples of the region $s_{\Lambda}>0$. Fig. 11 contains 2 separate instances of 4 phases meeting at a point as above, with the 4 phases falling into the classes labeled above; these points are labeled in both figures by the shaded circles.

It is the main result of this section and summarizes essentially all of the physics we are trying to describe.

We now describe the various phases of $S_{\text {eff }}$, which are summarized in Fig. 12

1. Commensurate, collinear, LRO: $\left\langle z^{\alpha}\right\rangle \neq 0,\langle\boldsymbol{\Lambda}\rangle=0$

This is the $(\pi, \pi)_{L R O}$ state with commensurate, collinear, magnetic LRO.

2. Commensurate, collinear, SRO: $\left\langle z^{\alpha}\right\rangle=0,\langle\boldsymbol{\Lambda}\rangle=0$

This is the $(\pi, \pi)_{S R O}$ "quantum-disordered" state with collinear spin correlations peaked at $(\pi, \pi)$. Its properties were described at length above. The compact $U(1)$ gauge force confines the $z^{\alpha}$ quanta. The spinless collective mode associated with the gauge fluctuations acquires a gap from monopole condensation, and the monopole Berry phases induce VBS order for odd $n_{b}$.

3. Incommensurate, coplanar, SRO: $\left\langle z^{\alpha}\right\rangle=0,\langle\boldsymbol{\Lambda}\rangle \neq 0$

This is the incommensurate phase with SRO at $(q, q)$ which we want to study. It is easy to see that condensation of $\boldsymbol{\Lambda}$ necessarily implies the appearance of incommensurate SRO: ignore fluctuations of $\boldsymbol{\Lambda}$ about $\langle\boldsymbol{\Lambda}\rangle$ and diagonalize the 
quadratic form controlling the $z^{\alpha}$ fluctuations; the minimum of the dispersion of the $z^{\alpha}$ quanta is at a non-zero wavevector

$$
\boldsymbol{k}_{0}=\left(\left\langle\Lambda_{x}\right\rangle,\left\langle\Lambda_{y}\right\rangle\right) / 2
$$

The spin structure factor will therefore have a maximum at an incommensurate wavevector. This phase also has a broken lattice rotation symmetry due to the choice of orientation in the $x-y$ plane made by $\boldsymbol{\Lambda}$ condensate, i.e., it has Isingnematic order.

The condensation of $\boldsymbol{\Lambda}$ also has a dramatic impact on the nature of the force between the massive $z^{\alpha}$ quanta. Detailed arguments have been presented by Fradkin and Shenker [79] that the condensation of a doubly charged Higgs scalar quenches the confining compact $U(1)$ gauge force in $2+1$ dimensions between singly charged particles. We can see this here from Eq. 60 by noticing that the condensation of $\boldsymbol{\Lambda}$ expels $A_{\mu}$ by the Meissner effect: consequently, monopoles in $A_{\mu}$ are connected by a flux tube whose action grows linearly with the separation between monopoles. The monopoles are therefore confined, and are unable to induce the confinement of the $z^{\alpha}$ quanta. From Eq. (60) we also see that once $\Lambda$ is condensed, the resulting theory for the spinons only has an effective $Z_{2}$ gauge invariance: Ref. [79] argued that there is an effective description of this free spinon phase in terms of a $Z_{2}$ gauge theory. The excitation structure is therefore very similar to that of the mean-field theory: spin-1/2, massive bosonic spinons and spinless collective modes which have a gap. The collective mode gap is present in this case even at $N=\infty$ and is associated with the condensation of $\boldsymbol{\Lambda}$.

This state is also 'topologically ordered'. We can see this by noticing [54, 55] that it carries stable point-like excitations which are $2 \pi$ vortices in either of $\Lambda_{x}$ or $\Lambda_{y}$. Because of the screening by the $A_{\mu}$ gauge field, the vortices carry a finite energy (this is analogous to the screening of supercurrents by the magnetic field around an Abrikosov vortex in a superconductor). Because the $\boldsymbol{\Lambda}$ carry charge -2 , the total $A_{\mu}$ flux trapped by a vortex is $\pi$. Thus the vortices are also stable to monopole tunneling events, which change the $A_{\mu}$ flux by integer multiples of $2 \pi$. A $z_{\alpha}$ spinon circumnavigating such a vortex would pick up an Aharanov-Bohm phase factor of $\pi$ (because the spinons have unit charge), and this is equivalent to the statement that the vortex and the spinon obey mutual 'semionic' statistics. All these characteristics identify the vortex excitation as one dubbed later [80] as the vison.

The vison also allows us to see the degeneracy of the gapped ground state on surfaces of non-trivial topology. We can insert a vison through any of the 'holes' in surface, and obtain a new candidate eigenstate. This eigenstate has an energy essentially degenerate with the ground state because the core of the vortex is within the hole, and so costs no energy. The 'far field' of the vison is within the system, but it costs negligible energy because the currents have been fully screened by $A_{\mu}$ in this region. Thus we obtain a factor of 2 increase in the degeneracy for every 'hole' in the surface (which is in turn related to the genus of the surface). The state so obtained is now referred to as a $Z_{2}$ spin liquid, and has been la- 
beled as such in the figures. As we noted above, the present theory only yields $Z_{2}$ spin liquids with Ising-nematic order, associated with broken symmetry of $90^{\circ}$ lattice rotations. We also note an elegant exactly solvable model described by Kitaev [81], which has spinon and vison excitations with the characteristics described above, but without the Ising-nematic order.

4. Incommensurate, coplanar, LRO: $\left\langle z^{\alpha}\right\rangle \neq 0,\langle\boldsymbol{\Lambda}\rangle \neq 0$

The condensation of the $z$ quanta at the wavevector $\boldsymbol{k}_{0}$ above leads to incommensurate LRO in the $(q, q)_{L R O}$ phase, with the spin condensate spiraling in the plane.

We also note a recent work [37, 82, 83] which has given a dual perspective on the above phases, including an efficient description of the phase transitions between them, and applied the results to experiments on $\kappa-(\mathrm{ET})_{2} \mathrm{Cu}_{2}(\mathrm{CN})_{3}$.

\section{$3 d$-wave superconductors}

In our discussion of phase transitions in insulators we found that the low-energy excitations near the critical point were linked in some way to the broken symmetry of the magnetically ordered state. In the models of Section 2.1 the low-energy excitations involved long wavelength fluctuations of the order parameter. In Section 2.2 the connection to the order parameter was more subtle but nevertheless present: the field $z_{\alpha}$ in Eq. (60) is a 'fraction' of the order parameter as indicated in (57), and the gauge field $A_{\mu}$ represents a non-coplanarity in the local order parameter orientation.

We will now move from insulators to the corresponding transitions in $d$-wave superconductors. Thus we will directly address the criticality of the magnetic QPT at $x=x_{s}$ in Fig.11. We will also consider the criticality of the 'remnant' Ising-nematic ordering at $x=x_{m}$ within the superconducting phase. A crucial property of $d$-wave superconductors is that they generically contain gapless, fermionic Bogoliubov excitations, as we will review below. These gapless excitations have a massless Dirac spectrum near isolated points in Brillouin zone. While these fermionic excitations are present in the non-critical $d$-wave superconductor, it is natural to ask whether they modify the theory of the QPT. Even though they may not be directly related to the order parameter, we can ask if the order parameter and fermionic excitations couple in interesting ways, and whether this coupling modifies the universality class of the transition. These questions will be answered in the following subsections.

We note that symmetry breaking transitions in graphene are also described by field theories similar to those discussed in this section [84 85].

\subsection{Dirac fermions}

We begin with a review of the standard BCS mean-field theory for a $d$-wave superconductor on the square lattice, with an eye towards identifying the fermionic 
Bogoliubov quasiparticle excitations. For now, we assume we are far from any QPT associated with SDW, Ising-nematic, or other broken symmetries. We consider the generalized Hamiltonian

$$
H_{t J}=\sum_{k} \varepsilon_{k} c_{k \alpha}^{\dagger} c_{k \alpha}+J_{1} \sum_{\langle i j\rangle} \boldsymbol{S}_{i} \cdot \boldsymbol{S}_{j}
$$

where $c_{j \alpha}$ is the annihilation operator for an electron on site $j$ with spin $\alpha=\uparrow, \downarrow$, $c_{k \alpha}$ is its Fourier transform to momentum space, $\varepsilon_{k}$ is the dispersion of the electrons (it is conventional to choose $\varepsilon_{k}=-2 t_{1}\left(\cos \left(k_{x}\right)+\cos \left(k_{y}\right)\right)-2 t_{2}\left(\cos \left(k_{x}+k_{y}\right)+\right.$ $\left.\cos \left(k_{x}-k_{y}\right)\right)-\mu$, with $t_{1,2}$ the first/second neighbor hopping and $\mu$ the chemical potential), and the $J_{1}$ term is the same as that in Eq. (15) with

$$
S_{j a}=\frac{1}{2} c_{j \alpha}^{\dagger} \sigma_{\alpha \beta}^{a} c_{j \beta}
$$

and $\sigma^{a}$ the Pauli matrices. We will consider the consequences of the further neighbor exchange interactions in (15) for the superconductor in Section 3.3.1 below. Applying the BCS mean-field decoupling to $H_{t J}$ we obtain the Bogoliubov Hamiltonian

$$
H_{B C S}=\sum_{k} \varepsilon_{k} c_{k \alpha}^{\dagger} c_{k \alpha}-\frac{J_{1}}{2} \sum_{j \mu} \Delta_{\mu}\left(c_{j \uparrow}^{\dagger} c_{j+\hat{\mu}, \downarrow}^{\dagger}-c_{j \downarrow}^{\dagger} c_{j+\hat{\mu}, \uparrow}^{\dagger}\right)+\text { h.c. }
$$

For a wide range of parameters, the ground state energy is optimized by a $d_{x^{2}-y^{2}}$ wavefunction for the Cooper pairs: this corresponds to the choice $\Delta_{x}=-\Delta_{y}=$ $\Delta_{x^{2}-y^{2}}$. The value of $\Delta_{x^{2}-y^{2}}$ is determined by minimizing the energy of the BCS state

$$
E_{B C S}=J_{1}\left|\Delta_{x^{2}-y^{2}}\right|^{2}-\int \frac{d^{2} k}{4 \pi^{2}}\left[E_{k}-\varepsilon_{k}\right]
$$

where the fermionic quasiparticle dispersion is

$$
E_{k}=\left[\varepsilon_{k}^{2}+\left|J_{1} \Delta_{x^{2}-y^{2}}\left(\cos k_{x}-\cos k_{y}\right)\right|^{2}\right]^{1 / 2} .
$$

The energy of the quasiparticles, $E_{k}$, vanishes at the four points $( \pm Q, \pm Q)$ at which $\varepsilon_{k}=0$. We are especially interested in the low-energy quasiparticles in the vicinity of these points, and so we perform a gradient expansion of $H_{B C S}$ near each of them. We label the points $\boldsymbol{Q}_{1}=(Q, Q), \boldsymbol{Q}_{2}=(-Q, Q), \boldsymbol{Q}_{3}=(-Q,-Q)$, $\boldsymbol{Q}_{4}=(Q,-Q)$ and write

$$
c_{j \alpha}=f_{1 \alpha}\left(\boldsymbol{r}_{j}\right) e^{i \boldsymbol{Q}_{1} \cdot \boldsymbol{r}_{j}}+f_{2 \alpha}\left(\boldsymbol{r}_{j}\right) e^{i \boldsymbol{Q}_{2} \cdot \boldsymbol{r}_{j}}+f_{3 \alpha}\left(\boldsymbol{r}_{j}\right) e^{i \boldsymbol{Q}_{3} \cdot \boldsymbol{r}_{j}}+f_{4 \alpha}\left(\boldsymbol{r}_{j}\right) e^{i \boldsymbol{Q}_{4} \cdot \boldsymbol{r}_{j}},
$$

while assuming that the $f_{1-4, \alpha}(\boldsymbol{r})$ are slowly varying functions of $\boldsymbol{r}$. We also introduce the bispinors $\Psi_{1}=\left(f_{1 \uparrow}, f_{3 \downarrow}^{\dagger}, f_{1 \downarrow},-f_{3 \uparrow}^{\dagger}\right)$, and $\Psi_{2}=\left(f_{2 \uparrow}, f_{4 \downarrow}^{\dagger}, f_{2 \downarrow},-f_{4 \uparrow}^{\dagger}\right)$, and then express $H_{B C S}$ in terms of $\Psi_{1,2}$ while performing a spatial gradient expansion. This yields the following effective action for the fermionic quasiparticles: 


$$
\begin{aligned}
\mathcal{S}_{\Psi}=\int d & d^{2} r\left[\Psi_{1}^{\dagger}\left(\partial_{\tau}-i \frac{v_{F}}{\sqrt{2}}\left(\partial_{x}+\partial_{y}\right) \tau^{z}-i \frac{v_{\Delta}}{\sqrt{2}}\left(-\partial_{x}+\partial_{y}\right) \tau^{x}\right) \Psi_{1}\right. \\
+ & \left.\Psi_{2}^{\dagger}\left(\partial_{\tau}-i \frac{v_{F}}{\sqrt{2}}\left(-\partial_{x}+\partial_{y}\right) \tau^{z}-i \frac{v_{\Delta}}{\sqrt{2}}\left(\partial_{x}+\partial_{y}\right) \tau^{x}\right) \Psi_{2}\right]
\end{aligned}
$$

where the $\tau^{x, z}$ are $4 \times 4$ matrices which are block diagonal, the blocks consisting of $2 \times 2$ Pauli matrices. The velocities $v_{F, \Delta}$ are given by the conical structure of $E_{k}$ near the $Q_{1-4}$ : we have $v_{F}=\left|\nabla_{k} \varepsilon_{k}\right|_{k=Q_{a}} \mid$ and $v_{\Delta}=\left|J_{1} \Delta_{x^{2}-y^{2}} \sqrt{2} \sin (Q)\right|$. In this limit, the energy of the $\Psi_{1}$ fermionic excitations is $E_{k}=\left(v_{F}^{2}\left(k_{x}+k_{y}\right)^{2} / 2+\right.$ $\left.v_{\Delta}^{2}\left(k_{x}-k_{y}\right)^{2} / 2\right)^{1 / 2}$ (and similarly for $\Psi_{2}$ ), which is the spectrum of massless Dirac fermions.

\subsection{Magnetic ordering}

We now focus attention on the QPT involving loss of magnetic ordering within the $d$-wave superconductor at $x=x_{s}$ in Fig. 1. As in Section 2, we have to now consider the fluctuations of the SDW order parameter. We discussed two routes to such a magnetic ordering transition in Section 2, one involving the vector SDW order parameter in Section 2.1. and the other involving the spinor $z_{\alpha}$ in Section 2.2 In principle, both routes also have to be considered in the $d$-wave superconductor. The choice between the two routes involves subtle questions on the nature of fractionalized excitations at intermediate scales which we will not explore further here. These questions were thoroughly addressed in Ref. [28] in the context of simple toy models: it was found that either route could apply, and the choice depended sensitively on microscopic details. In particular, it was found that among the fates of the nonmagnetic superconductor was that it acquired VBS or Ising-nematic ordering, as was found in the models explored in Section 2.2. This is part of the motivation for the expectation of such ordering in the regime $x_{s}<x<x_{m}$, as indicated in Fig. 1.

In the interests of brevity and simplicity, we will limit our discussion of the SDW ordering transition here to the vector formulation analogous to that in Section 2.1. We have full square lattice symmetry, and so allow for incommensurate SDW ordering similar to the $(q, q),(q,-q)$ and $(\pi, q),(q, \pi)$ states of Section 2.2. Because there are two distinct but degenerate ordering wavevectors, the complex order parameter $\Phi_{a}$ in Eq. (14) is now replaced by two complex order parameters $\Phi_{x a}$ and $\Phi_{y a}$ for orderings along $(\pi, q)$ and $(q, \pi)$ (the orderings along $(q, \pm q)$ can be treated similarly and we will not describe it explicitly). These order parameters are related to the spin operator by

$$
S_{a}(\boldsymbol{r})=\Phi_{x a} e^{i \boldsymbol{K}_{x} \cdot \boldsymbol{r}}+\Phi_{y a} e^{i \boldsymbol{K}_{y} \cdot \boldsymbol{r}}+\text { c.c. }
$$

where $\boldsymbol{K}_{x}=(q, \pi)$ and $\boldsymbol{K}_{y}=(\pi, q)$. As discussed below Eq. (14), depending upon the structure of the complex numbers $\Phi_{x a}, \Phi_{y a}$, the SDW ordering can be 


\begin{tabular}{c|ccccc} 
& $T_{x}$ & $T_{y}$ & $R$ & $I$ & $\mathcal{T}$ \\
\hline$\Phi_{x a}$ & $e^{i q} \Phi_{x a}$ & $-\Phi_{x a}$ & $\Phi_{y a}$ & $\Phi_{x a}^{*}$ & $-\Phi_{x a}$ \\
$\Phi_{y a}$ & $-\Phi_{y a}$ & $e^{i q} \Phi_{y a}$ & $\Phi_{x a}^{*}$ & $\Phi_{y a}^{*}$ & $-\Phi_{y a}$ \\
$\Psi_{1 \alpha}$ & $e^{i Q} \Psi_{1 \alpha}$ & $e^{i Q} \Psi_{1 \alpha}$ & $i \tau^{z} \Psi_{2 \alpha}$ & $\Psi_{2 \alpha}$ & $-\tau^{y} \Psi_{1 \alpha}$ \\
$\Psi_{2 \alpha}$ & $e^{-i Q} \Psi_{2 \alpha}$ & $e^{i Q} \Psi_{2 \alpha}$ & $-i \varepsilon_{\alpha \beta}\left[\Psi_{1 \beta}^{\dagger} \tau^{x}\right]^{T}$ & $\Psi_{1 \alpha}$ & $-\tau^{y} \Psi_{2 \alpha}$
\end{tabular}

Table 1 Transformations of the fields under operations which generate the symmetry group: $T_{x, y}=$ translation by a lattice spacing in the $x, y$ directions, $R=$ rotation about a lattice site by $90^{\circ}, I=$ reflection about the $y$ axis on a lattice site, and $\mathcal{T}=$ time reversal. The theory is also invariant under spin rotations, with $i$ a vector index and $\alpha, \beta$ spinor indices. We define $\mathcal{T}$ as an invariance of the imaginary time path integral, in which $\Phi_{1,2 i}^{*}$ transform as the complex conjugates of $\Phi_{1,2 i}$, while $\Psi_{1,2 \alpha}^{\dagger}$ are viewed as independent complex Grassman fields which transform as $\Psi_{1,2 \alpha}^{\dagger} \rightarrow \Psi_{1,2 \alpha}^{\dagger} \tau^{y}$.

either collinear (i.e., stripe-like) or spiral. Also, as in Eqs. (39) and (43), we can use these SDW order parameters to also define a subsidiary Ising-nematic order parameter

$$
\mathcal{I}=\left|\Phi_{x a}\right|^{2}-\left|\Phi_{y a}\right|^{2}
$$

to measure the breaking of $x \leftrightarrow y$ symmetry.

Symmetry considerations will play an important role in our analysis of the $\Phi_{x, y a}$ order parameters and their coupling to the Dirac fermions. In Table 1 we therefore present a table of transformations under important operations of the square lattice space group: these are easily deduced from the representations in Eq. (67) and (69).

The effective action for the SDW order parameters has a direct generalization from (14): it can be obtained by requiring invariance under the transformations in Table 1, and has many more allowed quartic nonlinearities [20]:

$$
\begin{array}{rl}
\mathcal{S}_{\Phi}=\int d^{2} & r d \tau\left[\left|\partial_{\tau} \Phi_{x a}\right|^{2}+c_{x}^{2}\left|\partial_{x} \Phi_{x a}\right|^{2}+c_{y}^{2}\left|\partial_{y} \Phi_{x a}\right|^{2}\right. \\
& +\left|\partial_{\tau} \Phi_{y a}\right|^{2}+c_{x}^{2}\left|\partial_{y} \Phi_{y a}\right|^{2}+c_{y}^{2}\left|\partial_{x} \Phi_{y a}\right|^{2}+s\left(\left|\Phi_{x a}\right|^{2}+\left|\Phi_{x a}\right|^{2}\right) \\
& +\frac{u_{1}}{2}\left[\left(\left|\Phi_{x a}\right|^{2}\right)^{2}+\left(\left|\Phi_{y a}\right|^{2}\right)^{2}\right]+\frac{u_{2}}{2}\left[\left|\left(\Phi_{x a}\right)^{2}\right|^{2}+\left|\left(\Phi_{y a}\right)^{2}\right|^{2}\right] \\
& \left.+w_{1}\left|\Phi_{x a}\right|^{2}\left|\Phi_{y a}\right|^{2}+w_{2}\left|\Phi_{x a} \Phi_{y a}\right|^{2}+w_{3}\left|\Phi_{x a} \Phi_{y a}^{*}\right|^{2}\right]
\end{array}
$$

Remarkably, a fairly complete 5-loop renormalization group analysis of this model has been carried out by De Prato et al. [86], and reliable information on its critical properties is now available.

(We note parenthetically that Eq. (71) concerns the theory of the transition at $x_{s}$ from an SDW ordered state to a $d$-wave superconductor with the full symmetry of the square lattice. However, as we have discussed in Section 1 and in the beginning 


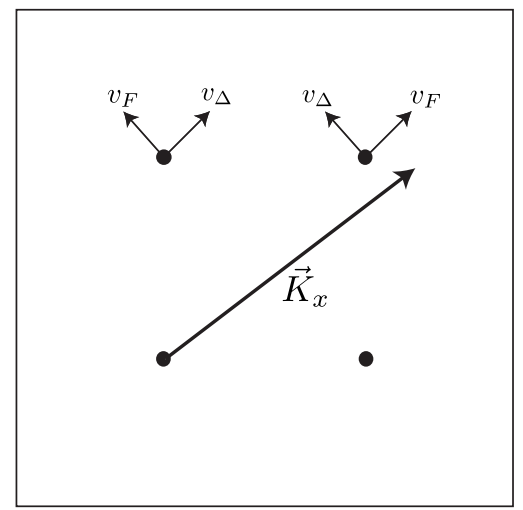

Fig. 13 The filled circles indicate the positions of the gapless Dirac fermions in the square lattice Brillouin zone: these are at wavevectors $\boldsymbol{Q}_{1,2,3,4}$. An SDW fluctuation scatters a fermion at one of the nodes by wavevector $\boldsymbol{K}_{x}$ to a generic point in the Brillouin zone. The final state of the fermion has a high energy, and so such processes are suppressed.

of Section 3 , there could be Ising nematic order in the regime $x_{s}<x<x_{m}$. In this case one of $\Phi_{x a}$ or $\Phi_{y a}$ orderings would be preferred, and we need only consider the critical fluctuations of this preferred component. The resulting action for this preferred component would then be identical to Eq. (14), with critical properties as in Ref. [53].)

Now we turn to the crucial issue of the coupling between the $\Phi_{x, y a}$ order parameter degrees of freedom in $\mathcal{S}_{\Phi}$ and the massless Dirac fermions $\Psi_{1,2}$ in Eq. 68.) Again a great deal follows purely from symmetry considerations. The simplest possible terms are cubic 'Yukawa' interaction terms like $\Phi_{x a} \Psi_{1}^{\dagger} \Psi_{2}$ etc. However, these are generically forbidden by translational invariance, or equivalently, momentum conservation. In particular, the transformation of the $\Phi_{x, y a}$ under translation by one lattice spacing follows from (69), while those of the $\Psi_{1,2}$ follow from (67). Unless the SDW ordering wavevectors $\boldsymbol{K}_{x, y}$ and the positions of the Dirac nodes $\boldsymbol{Q}_{1,2,3,4}$ satisfy certain commensurability conditions, the Yukawa coupling will not be invariant under this translation operation. This is illustrated schematically in Fig. 13 . The observed values of the wavevectors are not commensurate, and so we can safely neglect the Yukawa term.

The absence of the Yukawa coupling suggests that the fixed point theory describing the QPT at $x=x_{s}$ in the superconductor may be $\mathcal{S}_{\Phi}$ in Eq. (71) alone, i.e., the transition is in the same universality class as the insulator. However, to ensure this, we have to examine the influence of higher terms coupling the degrees of freedom of $\mathcal{S}_{\phi}$ and $\mathcal{S}_{\Psi}$. The simplest couplings not prohibited by translational invariance are associated with operators which are close to net zero momentum in both sectors. These are further constrained by the other square lattice space group operations in Table 1, requiring invariance under them shows that the simplest allowed terms are [87] 


$$
\begin{aligned}
& \mathcal{S}_{1}=\vartheta_{1} \int d \tau d^{2} r\left(\left|\Phi_{x a}\right|^{2}+\left|\Phi_{y a}\right|^{2}\right)\left(\Psi_{1}^{\dagger} \tau^{z} \Psi_{1}+\Psi_{2}^{\dagger} \tau^{z} \Psi_{2}\right) \\
& \mathcal{S}_{2}=\vartheta_{2} \int d \tau d^{2} r\left(\left|\Phi_{x a}\right|^{2}-\left|\Phi_{y a}\right|^{2}\right)\left(\Psi_{1}^{\dagger} \tau^{x} \Psi_{1}+\Psi_{2}^{\dagger} \tau^{x} \Psi_{2}\right)
\end{aligned}
$$

The first term is a fairly obvious 'density-density' coupling between the energies of the two systems. The second is more interesting: it involves the Ising nematic order $\mathcal{I}$, as measured in the order parameter sector by $[70$, and in the fermion sector by the bilinear shown above.

Now we can ask if the fixed point described by the decoupled theory $\mathcal{S}_{\Phi}+\mathcal{S}_{\Psi}$ is stable under the perturbations in $\mathcal{S}_{1}$ and $\mathcal{S}_{2}$. This involves a computation of the scaling dimensions of the couplings $\vartheta_{1,2}$ at the decoupled theory fixed point. These scaling dimensions were computed to 5-loop order in Ref. [87], and it was found that $\operatorname{dim}\left[\vartheta_{1}\right] \approx-1.0$, and $\operatorname{dim}\left[\vartheta_{2}\right] \approx-0.1$. Thus both couplings are irrelevant, and we can indeed finally conclude that the SDW onset transition is described by the same theory as in the insulator. However, the scaling dimension of the $\vartheta_{2}$ coupling is quite small, indicating that it will lead to appreciable effects. Thus we have demonstrated quite generally that it is the Ising-nematic order $\mathcal{I}$ which is most efficient in coupling the SDW order parameter fluctuations to the Dirac fermions. Note that $\mathcal{I}$ was not chosen by hand, but was selected by the theory among all other possible composite orders of the SDW field $\Phi_{x, y a}$. The near zero scaling dimension of $\vartheta_{2}$ implies that it will induce a linewidth $\sim T$ in the Dirac fermion spectrum. Moreover, because this broadening is mediated by $\mathcal{I}$, the broadening will be strongly anisotropic in space [88].

\subsection{Ising transitions}

Now we turn our attention to the vicinity of the point $x_{m}$ in Fig. 1 Although $x_{m}$ was defined in terms of the SDW transition in the metal at high magnetic fields, we have also argued in Section 1 and in the beginning of Section 3 that there can also be transitions associated with VBS or Ising nematic order near $x_{m}$ but within the superconducting phase at zero field. Strong evidence for a nematic order transition near $x_{m}$ has emerged in recent experiments [12,30,31].

This section will therefore consider the theory of Ising-nematic ordering within a $d$-wave superconductor. Unlike the situation in Section 3.2, we will find here that the order parameter and the Dirac fermions are strongly coupled, and the universality class of the transition is completely changed by the presence of the Dirac fermions. In Section 3.2 we found that although the fermions were moderately strongly coupled to the critical theory, they were ultimately reduced to spectators to the asymptotic critical behavior.

Before considering the Ising nematic transition, we will take a short detour in Section 3.3.1 and describe another Ising transition associated with the breaking of time-reversal symmetry in a $d$-wave superconductor. This leads to a model which 
has a somewhat simpler structure, and for which conventional renormalization group techniques work easily. We will return to Ising-nematic ordering in Section 3.3.2.

\subsubsection{Time-reversal symmetry breaking}

We will consider a simple model in which the pairing symmetry of the superconductor changes from $d_{x^{2}-y^{2}}$ to $d_{x^{2}-y^{2}} \pm i d_{x y}$. The choice of the phase between the two pairing components leads to a breaking of time-reversal symmetry. Studies of this transition were originally motivated by the cuprate phenomenology, but we will not explore this experimental connection here because the evidence has remained sparse.

The mean field theory of this transition can be explored entirely within the context of BCS theory, as we will review below. However, fluctuations about the BCS theory are strong, and lead to non-trivial critical behavior involving both the collective order parameter and the Bogoliubov fermions: this is probably the earliest known example [89,90] of the failure of BCS theory in two (or higher) dimensions in a superconducting ground state. At $T>0$, this failure broadens into the "quantum critical" region.

We extend $H_{t J}$ in Eq. 62 so that BCS mean-field theory permits a region with $d_{x y}$ superconductivity. It turns out that the frustrating interactions as in Eq. (15) are precisely those needed. With a $J_{2}$ interaction, Eq. 62) is modified to:

$$
\widetilde{H}_{t J}=\sum_{k} \varepsilon_{k} c_{k \sigma}^{\dagger} c_{k \sigma}+J_{1} \sum_{\langle i j\rangle} \boldsymbol{S}_{i} \cdot \mathbf{S}_{j}+J_{2} \sum_{\operatorname{nnn} i j} \boldsymbol{S}_{i} \cdot \boldsymbol{S}_{j} .
$$

We will follow the evolution of the ground state of $\widetilde{H}_{t J}$ as a function of $J_{2} / J_{1}$.

The mean-field Hamiltonian is now modified from Eq. 64, to

$$
\begin{aligned}
\widetilde{H}_{B C S}=\sum_{k} \varepsilon_{k} c_{k \sigma}^{\dagger} c_{k \sigma} & -\frac{J_{1}}{2} \sum_{j, \mu} \Delta_{\mu}\left(c_{j \uparrow}^{\dagger} c_{j+\hat{\mu}, \downarrow}^{\dagger}-c_{j \downarrow}^{\dagger} c_{j+\hat{\mu}, \uparrow}^{\dagger}\right)+\text { h.c. } \\
& -\frac{J_{2}}{2} \sum_{j, \nu}^{\prime} \Delta_{\nu}\left(c_{j \uparrow}^{\dagger} c_{j+\hat{\nu}, \downarrow}^{\dagger}-c_{j \downarrow}^{\dagger} c_{j+\hat{\nu}, \uparrow}^{\dagger}\right)+\text { h.c. }
\end{aligned}
$$

where the second summation over $\nu$ is along the diagonal neighbors $\hat{x}+\hat{y}$ and $-\hat{x}+\hat{y}$. To obtain $d_{x y}$ pairing along the diagonals, we choose $\Delta_{x+y}=-\Delta_{-x+y}=$ $\Delta_{x y}$. We summarize our choices for the spatial structure of the pairing amplitudes (which determine the Cooper pair wavefunction) in Fig. 14. The values of $\Delta_{x^{2}-y^{2}}$ and $\Delta_{x y}$ are to be determined by minimizing the ground state energy (generalizing Eq. 65)

$$
E_{B C S}=J_{1}\left|\Delta_{x^{2}-y^{2}}\right|^{2}+J_{2}\left|\Delta_{x y}\right|^{2}-\int \frac{d^{2} k}{4 \pi^{2}}\left[E_{k}-\varepsilon_{k}\right]
$$

where the quasiparticle dispersion is now (generalizing Eq. 666) 


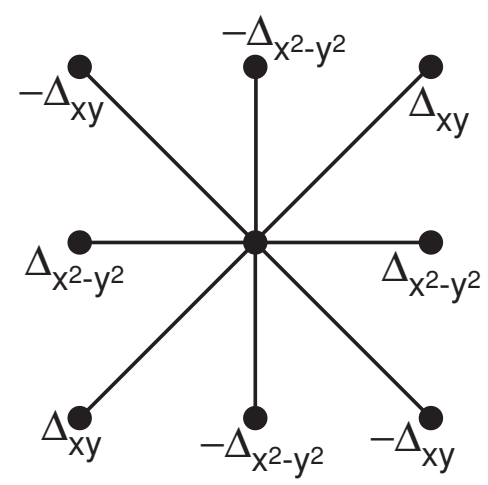

Fig. 14 Values of the pairing amplitudes, $-\left\langle c_{i \uparrow} c_{j \downarrow}-c_{i \downarrow} c_{j \uparrow}\right\rangle$ with $i$ the central site, and $j$ is one of its 8 nearest neighbors.

$$
E_{k}=\left[\varepsilon_{k}^{2}+\left|J_{1} \Delta_{x^{2}-y^{2}}\left(\cos k_{x}-\cos k_{y}\right)+2 J_{2} \Delta_{x y} \sin k_{x} \sin k_{y}\right|^{2}\right]^{1 / 2}
$$

Notice that the energy depends upon the relative phase of $\Delta_{x^{2}-y^{2}}$ and $\Delta_{x y}$ : this phase is therefore an observable property of the ground state.

It is a simple matter to numerically carry out the minimization of Eq. (76), and the results for a typical choice of parameters are shown in Fig. 15 as a function $J_{2} / J_{1}$. One of the two amplitudes $\Delta_{x^{2}-y^{2}}$ or $\Delta_{x y}$ is always non-zero and so the ground state is always superconducting. The transition from pure $d_{x^{2}-y^{2}}$ superconductivity to pure $d_{x y}$ superconductivity occurs via an intermediate phase in which both order parameters are non-zero. Furthermore, in this regime, their relative phase is found to be pinned to $\pm \pi / 2$, i.e.

$$
\arg \left(\Delta_{x y}\right)=\arg \left(\Delta_{x^{2}-y^{2}}\right) \pm \pi / 2
$$

The reason for this pinning can be intuitively seen from Eq. (76): only for these values of the relative phase does the equation $E_{k}=0$ never have a solution. In other words, the gapless nodal quasiparticles of the $d_{x^{2}-y^{2}}$ superconductor acquire a finite energy gap when a secondary pairing with relative phase $\pm \pi / 2$ develops. By a level repulsion picture, we can expect that gapping out the low-energy excitations should help lower the energy of the ground state. The intermediate phase obeying Eq. (77) is called a $d_{x^{2}-y^{2}}+i d_{x y}$ superconductor.

The choice of the sign in Eq. (77) leads to an overall two-fold degeneracy in the choice of the wavefunction for the $d_{x^{2}-y^{2}}+i d_{x y}$ superconductor. This choice is related to the breaking of time-reversal symmetry, and implies that the $d_{x^{2}-y^{2}}+i d_{x y}$ phase is characterized by the non-zero expectation value of a $Z_{2}$ Ising order parameter; the expectation value of this order vanishes in the two phases (the $d_{x^{2}-y^{2}}$ and $d_{x y}$ superconductors) on either side of the $d_{x^{2}-y^{2}}+i d_{x y}$ superconductor. As is conventional, we will represent the Ising order by a real scalar field $\phi$. Fluctuations of $\phi$ become critical near both of the phase boundaries in Fig. 15. As we will explain be- 


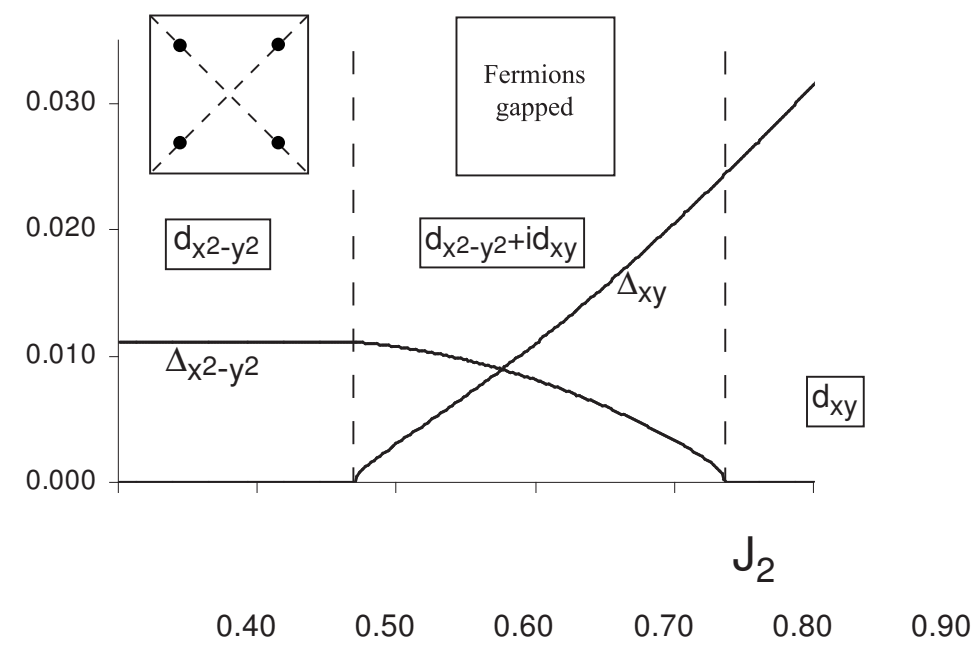

Fig. 15 BCS solution of the phenomenological Hamiltonian $\widetilde{H}_{t J}$ in Eq. 73 . Shown are the optimum values of the pairing amplitudes $\left|\Delta_{x^{2}-y^{2}}\right|$ and $\left|\Delta_{x y}\right|$ as a function of $J_{2}$ for $t_{1}=1$, $t_{2}=-0.25, \mu=-1.25$, and $J_{1}$ fixed at $J_{1}=0.4$. The relative phase of the pairing amplitudes was always found to obey Eq. (77). The dashed lines denote locations of phase transitions between $d_{x^{2}-y^{2}}, d_{x^{2}-y^{2}}+i d_{x y}$, and $d_{x y}$ superconductors. The pairing amplitudes vanishes linearly at the first transition corresponding to the exponent $\beta_{B C S}=1$ in Eq. 80. The Brillouin zone location of the gapless Dirac points in the $d_{x^{2}-y^{2}}$ superconductor is indicated by filled circles. For the dispersion $\varepsilon_{k}$ appropriate to the cuprates, the $d_{x y}$ superconductor is fully gapped, and so the second transition is ordinary Ising.

low, the critical theory of the $d_{x^{2}-y^{2}}$ to $d_{x^{2}-y^{2}}+i d_{x y}$ transition is not the usual $\phi^{4}$ field theory which describes the ordinary Ising transition in three spacetime dimensions. (For the dispersion $\varepsilon_{k}$ appropriate to the cuprates, the $d_{x y}$ superconductor is fully gapped, and so the $d_{x^{2}-y^{2}}+i d_{x y}$ to $d_{x y}$ transition in Fig. 15 will be ordinary Ising.)

Near the phase boundary from $d_{x^{2}-y^{2}}$ to $d_{x^{2}-y^{2}}+i d_{x y}$ superconductivity it is clear that we can identify

$$
\phi=i \Delta_{x y}
$$

(in the gauge where $\Delta_{x^{2}-y^{2}}$ is real). We can now expand $E_{B C S}$ in Eq. 75 for small $\phi$ (with $\Delta_{x^{2}-y^{2}}$ finite) and find a series with the structure [91 92]

$$
E_{B C S}=E_{0}+s \phi^{2}+v|\phi|^{3}+\ldots,
$$

where $s, v$ are coefficients and the ellipses represent regular higher order terms in even powers of $\phi ; s$ can have either sign, whereas $v$ is always positive. Notice the non-analytic $|\phi|^{3}$ term that appears in the BCS theory - this arises from an infrared singularity in the integral in Eq. (75) over $E_{k}$ at the four nodal points of the $d_{x^{2}-y^{2}}$ superconductor, and is a preliminary indication that the transition differs from that 
in the ordinary Ising model, and that the Dirac fermions play a central role. We can optimize $\phi$ by minimizing $E_{B C S}$ in Eq. $79 p$ - this shows that $\langle\phi\rangle=0$ for $s>0$, and $\langle\phi\rangle \neq 0$ for $s<0$. So $s \sim\left(J_{2} / J_{1}\right)_{c}-J_{2} / J_{1}$ where $\left(J_{2} / J_{1}\right)_{c}$ is the first critical value in Fig. 15. Near this critical point, we find

$$
\langle\phi\rangle \sim\left(s_{c}-s\right)^{\beta}
$$

where we have allowed for the fact that fluctuation corrections will shift the critical point from $s=0$ to $s=s_{c}$. The present BCS theory yields the exponent $\beta_{B C S}=1$; this differs from the usual mean-field exponent $\beta_{M F}=1 / 2$, and this is of course due to the non-analytic $|\phi|^{3}$ term in Eq. 799.

We have laid much of the ground work for the required field theory of the onset of $d_{x y}$ order in Section 3.2. In addition to the order parameter $\phi$, the field theory should also involve the low-energy nodal fermions of the $d_{x^{2}-y^{2}}$ superconductor, as described by $\mathcal{S}_{\Psi}$ in Eq. (68). For the $\phi$ fluctuations, we write down the usual terms permitted near a phase transition with Ising symmetry, and similar to those in Eq. (12):

$$
\mathcal{S}_{\phi}=\int d^{2} r d \tau\left[\frac{1}{2}\left(\left(\partial_{\tau} \phi\right)^{2}+c^{2}\left(\partial_{x} \phi\right)^{2}+c^{2}\left(\partial_{y} \phi\right)^{2}+s \phi^{2}\right)+\frac{u}{24} \phi^{4}\right] .
$$

Note that, unlike Eq. (79), we do not have any non-analytic $|\phi|^{3}$ terms in the action: this is because we have not integrated out the low-energy Dirac fermions, and the terms in Eq. (81) are viewed as arising from high-energy fermions away from the nodal points. Finally, we need to couple the $\phi$ and $\Psi_{1,2}$ excitations. Their coupling is already contained in the last term in Eq. (74): expressing this in terms of the $\Psi_{1,2}$ fermions using Eq. 67) we obtain

$$
\mathcal{S}_{\Psi \phi}=\vartheta_{x y} \int d^{2} r d \tau\left[\phi\left(\Psi_{1}^{\dagger} \tau^{y} \Psi_{1}-\Psi_{2}^{\dagger} \tau^{y} \Psi_{2}\right)\right],
$$

where $\vartheta_{x y}$ is a coupling constant. This coupling also has been obtained by symmetry considerations, by examining invariants under the transformations of Table 1 The partition function of the full theory is now

$$
\mathcal{Z}=\int \mathcal{D} \phi \mathcal{D} \Psi_{1} \mathcal{D} \Psi_{2} \exp \left(-\mathcal{S}_{\Psi}-\mathcal{S}_{\phi}-\mathcal{S}_{\Psi \phi}\right),
$$

where $\mathcal{S}_{\Psi}$ was in Eq. (68). It can now be checked that if we integrate out the $\Psi_{1,2}$ fermions for a spacetime independent $\phi$, we do indeed obtain a $|\phi|^{3}$ term in the effective potential for $\phi$.

We begin our analysis of $\mathcal{Z}$ in Eq. 83 by following the procedure of Section 3.2 . Assume that the transition is described by a fixed point with $\vartheta_{x y}=0$ : then as in Section 3.2 the theory for the transition would be the ordinary $\phi^{4}$ field theory $\mathcal{S}_{\phi}$, and the nodal fermions would again be innocent spectators. The scaling dimension of $\phi$ at such a fixed point is $\left(1+\eta_{I}\right) / 2$ (where $\eta_{I}$ is the anomalous order parameter exponent at the critical point of the ordinary three dimensional Ising model), while 
that of $\Psi_{1,2}$ is 1 . Consequently, the scaling dimension of $\vartheta_{x y}$ is $\left(1-\eta_{I}\right) / 2>0$. This positive scaling dimension implies that $\vartheta_{x y}$ is relevant and the $\vartheta_{x y}=0$ fixed point is unstable: the Dirac fermions are fully involved in the critical theory.

Determining the correct critical behavior now requires a full renormalization group analysis of $\mathcal{Z}$. This has been described in some detail in Ref. [90], and we will not reproduce the details here. The main result we need for our purposes is that couplings $\vartheta_{x y}, u, v_{F} / c$ and $v_{\Delta} / c$ all reach non-zero fixed point values which define a critical point in a new universality class. These fixed point values, and the corresponding critical exponents, can be determined in expansions in either $(3-d)$ [89. 90] (where $d$ is the spatial dimensionality) or $1 / N$ [93] (where $N$ is the number of fermion species). An important simplifying feature here is that the fixed point is actually relativistically invariant. Indeed the fixed point has the structure of the so-called Higgs-Yukawa model which has been studied extensively in the particle physics literature [94] in a different physical context: quantum Monte Carlo simulation of this model also exist [95], and provide probably the most accurate estimate of the exponents.

The non-trivial fixed point has strong implications for the correlations of the Bogoliubov fermions. The fermion correlation function $G_{1}=\left\langle\Psi_{1} \Psi_{1}^{\dagger}\right\rangle$ obeys

$$
G_{1}(k, \omega)=\frac{\omega+v_{F} k_{x} \tau^{z}+v_{\Delta} \tau^{x}}{\left(v_{F}^{2} k_{x}^{2}+v_{\Delta}^{2} k_{y}^{2}-\omega^{2}\right)^{\left(1-\eta_{f}\right) / 2}}
$$

at low frequencies for $s \geq s_{c}$. Away from the critical point in the $d_{x^{2}-y^{2}}$ superconductor with $s>s_{c}$, Eq (84) holds with $\eta_{f}=0$, and this is the BCS result, with sharp quasi-particle poles in the Green's function. At the critical point $s=s_{c}$ Eq. (84) holds with the fixed point values for the velocities (which satisfy $v_{F}=v_{\Delta}=c$ ) and with the anomalous dimension $\eta_{f} \neq 0$ - the $(3-d)$ expansion [89] estimate is $\eta_{f} \approx(3-d) / 14$, and the $1 / N$ expansion estimate [93] is $\eta_{f} \approx 1 /\left(3 \pi^{2} N\right)$, with $N=2$. This is clearly non-BCS behavior, and the fermionic quasiparticle pole in the spectral function has been replaced by a branch-cut representing the continuum of critical excitations. The corrections to BCS extend also to correlations of the Ising order $\phi$ : its expectation value vanishes as Eq. (80) with the Monte Carlo estimate $\beta \approx 0.877$ [95]. The critical point correlators of $\phi$ have the anomalous dimension $\eta \approx 0.754$ [95], which is clearly different from the very small value of the exponent $\eta_{I}$ at the unstable $\vartheta_{x y}=0$ fixed point. The value of $\beta$ is related to $\eta$ by the usual scaling law $\beta=(1+\eta) \nu / 2$, with $\nu \approx 1.00$ the correlation length exponent (which also differs from the exponent $\nu_{I}$ of the Ising model).

\subsubsection{Nematic ordering}

We now turn, as promised, to the case of Ising-nematic ordering within the $d$-wave superconductor at $x=x_{m}$.

The ingredients of such an ordering are actually already present in our simple review of BCS theory in Section 3.1 In Eq. [64, we introduce 2 variational pairing 


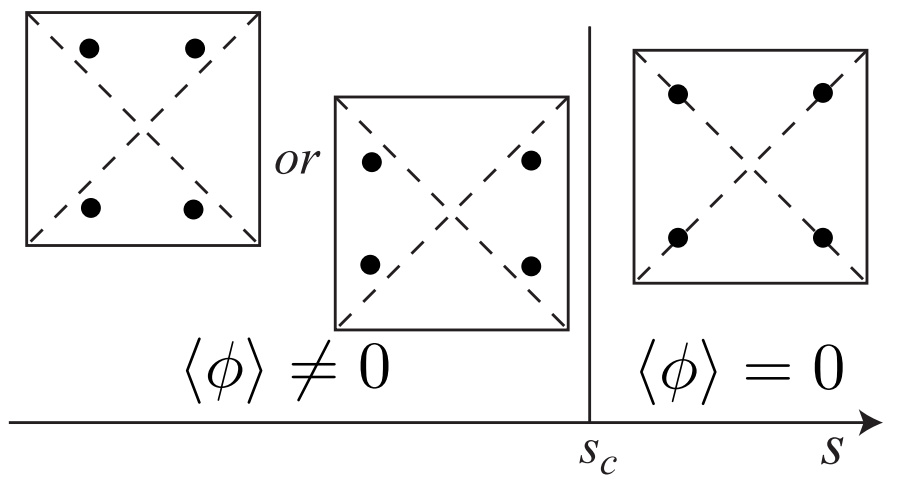

Fig. 16 Phase diagram of Ising nematic ordering in a $d$-wave superconductor as a function of the coupling $s$ in $\mathcal{S}_{\phi}$. The filled circles indicate the location of the gapless fermionic excitations in the Brillouin zone. The two choices for $s<s_{C}$ are selected by the sign of $\langle\phi\rangle$.

amplitudes $\Delta_{x}$ and $\Delta_{y}$. Subsequently, we assumed that the minimization of the energy led to a solution with $d_{x^{2}-y^{2}}$ pairing symmetry with $\Delta_{x}=-\Delta_{y}=\Delta_{x^{2}-y^{2}}$. However, it is possible that upon including the full details of the microscopic interactions we are led to a minimum where the optimal solution also has a small amount of $s$-wave pairing. Then $\left|\Delta_{x}\right| \neq\left|\Delta_{y}\right|$, and we would expect all physical properties to have distinct dependencies on the $x$ and $y$ coordinates. So, as in Eqs. (39), (43) and (70), we can define the Ising-nematic order parameter by

$$
\mathcal{I}=\left|\Delta_{x}\right|^{2}-\left|\Delta_{y}\right|^{2} .
$$

The derivation of the field theory for this transition follows closely our presentation in Section 3.3.1 We allow for small Ising-nematic ordering by introducing a scalar field $\phi$ and writing

$$
\Delta_{x}=\Delta_{x^{2}-y^{2}}+\phi \quad ; \quad \Delta_{y}=-\Delta_{x^{2}-y^{2}}+\phi ;
$$

note that $\mathcal{I} \propto \phi$. The evolution of the Dirac fermion spectrum under such a change is indicated in Fig. 16. We now develop an effective action for $\phi$ and the Dirac fermions $\Psi_{1,2}$. The result is essentially identical to that in Section 3.3.1 apart from a change in the structure of the Yukawa coupling. Thus we obtain a theory $\mathcal{S}_{\Psi}+\mathcal{S}_{\phi}+\overline{\mathcal{S}}_{\Psi \phi}$, defined by Eqs. (68) and (81), and where Eq. (82) is now replaced by

$$
\overline{\mathcal{S}}_{\Psi \phi}=\vartheta_{I} \int d^{2} r d \tau\left[\phi\left(\Psi_{1}^{\dagger} \tau^{x} \Psi_{1}+\Psi_{2}^{\dagger} \tau^{x} \Psi_{2}\right)\right] .
$$

Not surprisingly, the fermion bilinear coupling to the nematic order parameter $\phi$ is identical to that in Eq. (72), as is expected from the transformations of Table 1.

The seemingly innocuous change between Eqs. (82) and (87) however has strong consequences. This is partly linked to the fact with $\overline{\mathcal{S}}_{\Psi \phi}$ cannot be relativistically invariant even after all velocities are adjusted to be equal. A weak-coupling renor- 
malization group analysis in powers of the coupling $\vartheta_{I}$ was performed in $(3-d)$ dimensions in Refs. [89,90], and led to flows to strong coupling with no accessible fixed point: thus no firm conclusions on the nature of the critical theory were drawn.

This problem remained unsolved until the recent works of Refs. [88, 96]. It is essential that the coupling $\vartheta_{I}$ not be used as a perturbative expansion parameter. This is because it leads to strongly non-analytic changes in the structure of the $\phi$ propagator, which have to be included at all stages. In a model with $N$ fermion flavors, the $1 / N$ expansion does avoid any expansion in $\vartheta_{I}$. The renormalization group analysis has to be carried out within the context of the $1 / N$ expansion, and this involves some rather technical analysis which is explained in Ref. [96]. In the end, an asymptotically exact description of the vicinity of the critical point was obtained. It was found that the velocity ratio $v_{F} / v_{\Delta}$ diverged logarithmically with energy scale, leading to strongly anisotropic 'arc-like' spectra for the Dirac fermions. Associated singularities in the thermal conductivity have also been computed [97].

\section{Metals}

We finally turn to the transition in the metal at $x_{m}$, which anchored our discussion of the cuprate phase diagram in Section 1 This controls the high field transition line in Fig. 1 between the large Fermi surface and small Fermi pocket states. We also argued that this transition was a key ingredient in a theory of the strange metal.

In addition to the order parameter ingredients we met in Section 2 , we now have to also account for fermion excitations as in Section 3 In Section 3 the fermionic excitations had vanishing energy only at isolated nodal points in the Brillouin zone: see Fig.13. In the present section we are dealing with metals, which have fermionic excitations with vanishing energy along an entire line in the Brillouin zone. Thus we can expect them to have an even stronger effect on the critical theory. This will indeed be the case, and we will be led to problems with a far more complex structure. Unlike the situation in insulators and $d$-wave superconductors, many basic issues associated with ordering transitions in two dimensional metals have not been fully resolved. The problem remains one of active research and is being addressed by many different approaches.

As discussed in Sections 2 and 3.2 , we can describe magnetic ordering by using either vector or spinor variables for the order parameter, and these lead to very different phases and critical points. For metals, the relationship between these two approaches, and their distinct physical properties have been described recently in Ref. [15]. The spinor route is more 'exotic' and leads to intermediate non-Fermi liquid critical phases between the small and large Fermi surface Fermi liquid phases. These intermediate critical phases could well be important for the experiments and for Fig. 1. but we will not describe them here. We will limit our present discussion to the more conventional vector mode description of the SDW ordering transition.

In recent papers [98 99] Metlitski and the author have argued that the problem of symmetry breaking transitions in two-dimensional metals is strongly coupled, and 


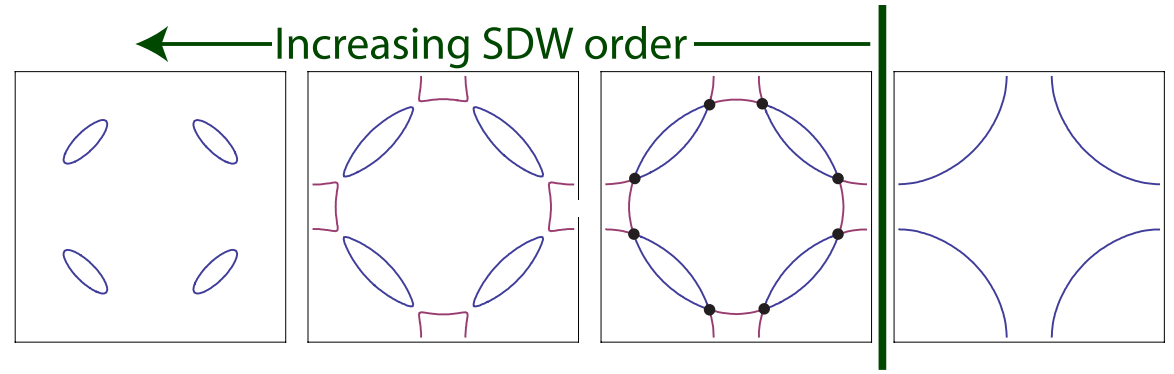

Fig. 17 Evolution of the Fermi surface of the hole doped cuprates in a conventional SDW theory [8] as a function of the magnitude of the SDW order $\left|\varphi_{a}\right|$, obtained from Eq. 90]. The right panel is the large Fermi surface state with no SDW order, with states contiguous to $\boldsymbol{k}=0$ occupied by electrons. The "hot spots" are indicated by the filled circles in the second panel from the right. The onset of SDW order induces the formation of electron and hole pockets (the hole pockets are the ones intersecting the diagonals of the Brillouin zone). With further increase of $\left|\varphi_{a}\right|$, the electron pockets disappear and only hole pockets remain (the converse happens in the last step for the electron-doped cuprates).

proposed field theories and scaling structures for the vicinity of the critical point. Here we will be satisfied with a simple description of the effective action and its mean field theory [100]: the reader is referred to the recent papers [98 99] for further analyses.

As in Section 3 , let us begin by a description of the non-critical fermionic sector, before its coupling to the order parameter fluctuations. We use the band structure describing the cuprates in the over-doped region, well away from the Mott insulator. Here the electrons $c_{\boldsymbol{k} \alpha}$ are described by the kinetic energy in Eq. [62, which we write in the following action

$$
\mathcal{S}_{c}=\int d \tau \sum_{\boldsymbol{k}} c_{k \alpha}^{\dagger}\left(\frac{\partial}{\partial \tau}+\varepsilon_{k}\right) c_{k \alpha} .
$$

This band structure leads to the Fermi surface shown in the right-most panel of Fig. 17, and also later in Fig. 18

\subsection{SDW ordering}

As discussed in Section 3, we must now couple the fermions of Eq. (88) to the bosonic modes associated with the SDW ordering transition. As noted above, we will use the more conventional vector mode description of the SDW ordering transition using the order parameters in Eq. (69). The analog of the coupling in Eq. (82) now leads to the interaction term 


$$
\mathcal{S}_{c \Phi}=\int d \tau \sum_{\boldsymbol{k}, \boldsymbol{q}} \Phi_{x a}(\boldsymbol{q}) c_{\boldsymbol{k}+\boldsymbol{K}_{x}+\boldsymbol{q}, \alpha}^{\dagger} \sigma_{\alpha \beta}^{a} c_{k \beta}+\text { c.c. }+x \rightarrow y,
$$

where $\boldsymbol{q}$ is a small momentum associated with a long-wavelength SDW fluctuation, while the sum over the momentum $\boldsymbol{k}$ extends over the entire Brillouin zone. The complete theory for the SDW transition is now contained, in principle, in $\mathcal{S}_{c}+$ $\mathcal{S}_{c \Phi}+\mathcal{S}_{\Phi}$, with $\mathcal{S}_{\Phi}$ contained in Eq. (71).

Let us consider the mean-field predictions of this theory in the SDW ordered state. For simplicity, we consider ordering at $\boldsymbol{K}=(\pi, \pi)$, in which case $\Phi_{x a}$ and $\Phi_{y a}$ both reduce to the Néel order $\varphi_{a}$ in Eq. (12). In the state with SDW order, we can take $\varphi_{a}=(0,0, \varphi)$ a constant. Then $\mathcal{S}_{c}+\mathcal{S}_{c \Phi}$ is a bilinear in the fermions and can be diagonalized to yield a fermion band structure (the analog of Eq. 667)

$$
E_{k}=\frac{\varepsilon_{k}+\varepsilon_{\boldsymbol{k}+\boldsymbol{K}}}{2} \pm\left(\left(\frac{\varepsilon_{k}+\varepsilon_{\boldsymbol{k}+\boldsymbol{K}}}{2}\right)^{2}+\varphi^{2}\right)^{1 / 2} .
$$

Filling the lowest energy bands of this dispersion leads to the Fermi surface structure [8] shown in Fig. 17] The second panel from the right shows the Fermi surface obtained by translating the original Fermi surface by $\boldsymbol{K}$, and the remaining panels show the consequences of mixing between the states at momentum $\boldsymbol{k}$ and $\boldsymbol{k}+\boldsymbol{K}$. Note that the Fermi surface has split apart into "small" electron and hole pockets, as discussed in Section 1

Let us now attempt to move beyond this simple mean field theory. As written, the action $\mathcal{S}_{c}+\mathcal{S}_{c \Phi}+\mathcal{S}_{\Phi}$ is not conducive to a field-theoretic analysis: this is mainly because the sum over $\boldsymbol{k}$ in Eq. (89) extends over the entire Brillouin zone, and there are low-energy fermionic excitations along an entire line of $k$ close the the Fermi surface. However, one simplifying feature here is that most of these low-energy fermions do not couple efficiently to the SDW order parameter, and their situation is similar to the fate of the Dirac fermions illustrated in Fig. 13 - upon scattering with the wavevector $\boldsymbol{K}$, they end up at generic points in the Brillouin zone at which there are only high-energy fermionic states. There are now 8 special "hot spots" on the Fermi surface which do connect via the wavevector $\boldsymbol{K}$ to other spots directly on the Fermi surface: these are illustrated in Fig. 17. These "hot spots" are thus similar to the Dirac hot spots we met in Section 3.3 upon considering Ising transitions with a zero-momentum order parameter in a $d$-wave superconductor. However the present situation is more complex because we also have "cold lines" of zero energy fermionic excitations coming into the hot spots.

A successful theory of the fermionic hot spots was reviewed in Section 3.3 A natural idea is to apply the same approach to the present situation with fermionic hot spots and cold lines. This leads to a problem of considerably complexity, which remains strongly coupled even within the context of the $1 / N$ expansion: see Refs. [99.101] for further details. 


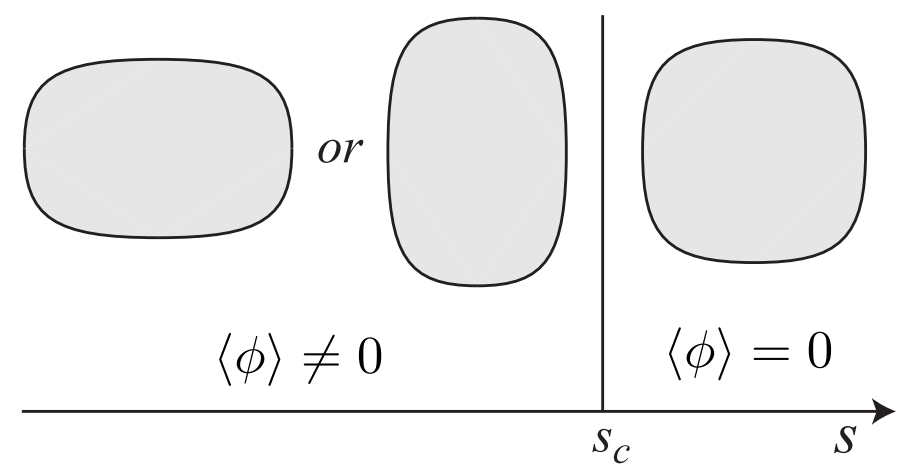

Fig. 18 Phase diagram of Ising nematic ordering in a metal as a function of the coupling $s$ in $\mathcal{S}_{\phi}$. The Fermi surface for $s>0$ is the same as that in the right-most panel of Fig. 17 but with the $\boldsymbol{k}=0$ point shifted from the center to the edge of the Brillouin zone. The interior regions are the occupied hole (or empty electron) states. The choice between the two quadrapolar distortions of the Fermi surface is determined by the sign of $\langle\phi\rangle$.

\subsection{Nematic ordering}

For completeness, we also consider the case of the Ising-nematic ordering in the presence of the large Fermi surface metal. Then we will have an Ising order parameter represented by the real scalar field $\phi$, which is described as before by Eq. (81). Its coupling to the electrons can be deduced by symmetry considerations, and the most natural coupling (the analog of Eqs. (87) and (89p) is

$$
\mathcal{S}_{c \phi}=\int d \tau \sum_{\boldsymbol{k}, \boldsymbol{q}}\left(\cos k_{x}-\cos k_{y}\right) \phi(\boldsymbol{q}) c_{\boldsymbol{k}+\boldsymbol{q} / 2, \alpha}^{\dagger} c_{\boldsymbol{k}-\boldsymbol{q} / 2, \alpha}
$$

The momentum dependent form factor is the simplest choice with changes sign under $x \leftrightarrow y$, as is required by the symmetry properties of $\phi$. Again, the sum over $\boldsymbol{q}$ is over small momenta, while that over $\boldsymbol{k}$ extends over the entire Brillouin zone. The theory for the nematic ordering transition is now described by $\mathcal{S}_{c}+\mathcal{S}_{\phi}+\mathcal{S}_{c \phi}$. The evolution of the Fermi surface as a function of the Ising coupling in $\mathcal{S}_{\phi}$ is shown in Fig. 18 .

Note that Eq. 91) does not have any large momentum transfer associated with $\boldsymbol{K}$. Consequently, at any generic point on the Fermi surface, there can be scattering to other nearby low-energy fermionic excitations by long wavelength modes of $\phi$. In other words, the entire Fermi surface is "hot". Thus we are faced with a third case of a "hot line" of fermions coupled to the critical order parameter mode of the transition. This case has been analyzed in Ref. [98], where it is proposed that the critical point is actually described by an infinite number of $2+1$ dimensional field theories, labeled by points on the Fermi surface. The reader is referred to Ref. [98] for further results on this complex problem - a review of the main results appears in Ref. [102]. 


\section{Acknowledgements}

I thank Eun Gook Moon for valuable comments on the manuscript and for a collaboration [3] which led to Fig. 1] R. Fernandes, J. Flouquet, G. Knebel, and J. Schmalian, for providing the plots shown in Fig. 2, C. Ruegg for the plot shown in Fig. 7. and the participants of the schools for their interest, and for stimulating discussions. This research was supported by the National Science Foundation under grant DMR-0757145, by the FQXi foundation, and by a MURI grant from AFOSR.

\section{References}

1. J. G. Bednorz and K. A. Müller, Possible high $T_{c}$ superconductivity in the Ba-La-Cu-O syatem, Z. Phys. B 64, 188 (1986).

2. N. Doiron-Leyraud, C. Proust, D. LeBoeuf, J. Levallois, J.-B. Bonnemaison, R. Liang, D. A. Bonn, W. N. Hardy, and L. Taillefer, Quantum oscillations and the Fermi surface in an underdoped high- $T_{c}$ superconductor, Nature 447, 565 (2007).

3. E. G. Moon and S. Sachdev, Competition between spin density wave order and superconductivity in the underdoped cuprates, Phys. Rev. B 80, 035117 (2009).

4. S. Sachdev, Where is the quantum critical point in the cuprate superconductors?, Physica status solidi (b) 247, 537 (2010).

5. S. Sachdev, Quantum criticality and the phase diagram of the cuprates, 9th International Conference on Materials and Mechanisms of Superconductivity, Tokyo, Sep 7-12, 2009, Physica C 470, S4 (2010).

6. Y. Qi and S. Sachdev, Effective theory of Fermi pockets in fluctuating antiferromagnets, Phys. Rev. B 81, 115129 (2010).

7. E. G. Moon and S. Sachdev, The underdoped cuprates as fractionalized Fermi liquids: transition to superconductivity, Phys. Rev. B 83, 224508 (2011).

8. S. Sachdev, A. V. Chubukov, and A. Sokol, Crossover and scaling in a nearly antiferromagnetic Fermi liquid in two dimensions, Phys. Rev. B 51, 14874 (1995).

9. D. LeBoeuf, N. Doiron-Leyraud, J. Levallois, R. Daou, J.-B. Bonnemaison, N. E. Hussey, L. Balicas, B. J. Ramshaw, R. Liang, D. A. Bonn, W. N. Hardy, S. Adachi, C. Proust, and L. Taillefer, Electron pockets in the Fermi surface of hole-doped high- $T_{c}$ superconductors, Nature 450, 533 (2007).

10. S. E. Sebastian, N. Harrison, P. A. Goddard, M. M. Altarawneh, C. H. Mielke, Ruixing Liang, D. A. Bonn, W. N. Hardy, O. K. Andersen, and G. G. Lonzarich, Compensated electron and hole pockets in an underdoped high- $T_{c}$ superconductor, Phys. Rev. B 81, 214524 (2010).

11. R. Daou, N. Doiron-Leyraud, D. LeBoeuf, S. Y. Li, F. Laliberté, O. Cyr-Choinière, Y. J. Jo, L. Balicas, J.-Q. Yan, J.-S. Zhou, J. B. Goodenough, and L. Taillefer, Linear temperature dependence of resistivity and change in the Fermi surface at the pseudogap critical point of a high- $T_{c}$ superconductor, Nature Physics 5, 31 (2009).

12. R. Daou, J. Chang, D. LeBoeuf, O. Cyr-Choiniere, F. Laliberte, N. Doiron-Leyraud, B. J. Ramshaw, Ruixing Liang, D. A. Bonn, W. N. Hardy, and L. Taillefer, Broken rotational symmetry in the pseudogap phase of a high-Tc superconductor, Nature 463, 519 (2010).

13. T. Helm, M. V. Kartsovnik, M. Bartkowiak, N. Bittner, M. Lambacher, A. Erb, J. Wosnitza, and R. Gross, Evolution of the Fermi Surface of the Electron-Doped High-Temperature Superconductor $\mathrm{Nd}_{2-x} \mathrm{Ce}_{x} \mathrm{CuO}_{4}$ Revealed by Shubnikov-de Haas Oscillations, Phys. Rev. Lett. 103, 157002 (2009).

14. T. Helm, M. V. Kartsovnik, I. Sheikin, M. Bartkowiak, F. Wolff-Fabris, N. Bittner, W. Biberacher, M. Lambacher, A. Erb, J. Wosnitza, and R. Gross, Magnetic Breakdown in the Electron-Doped Cuprate Superconductor $\mathrm{Nd}_{2-x} \mathrm{Ce}_{x} \mathrm{CuO}_{4}$ : The Reconstructed Fermi Surface Survives in the Strongly Overdoped Regime, Phys. Rev. Lett. 105, 247002 (2010). 
15. S. Sachdev, M. A. Metlitski, Y. Qi, and C. Xu, Fluctuating spin density waves in metals, Phys. Rev. B 80, 155129 (2009).

16. D. J. Scalapino, The case for $d_{x^{2}-y^{2}}$ pairing in the cuprate superconductors, Phys. Rep. 250, 329 (1995).

17. Ar. Abanov, A. V. Chubukov, and J. Schmalian, Quantum-critical theory of the spin-fermion model and its application to cuprates: normal state analysis, Adv. Phys. 52, 119 (2003).

18. V. Galitski and S. Sachdev, Paired electron pockets in the hole-doped cuprates, Phys. Rev. B 79, 134512 (2009).

19. M. Kato and K. Machida, Superconductivity and spin-density waves: Application to heavyfermion materials, Phys. Rev. B 37, 1510 (1988).

20. E. Demler, S. Sachdev, and Y. Zhang, Spin-Ordering Quantum Transitions of Superconductors in a Magnetic Field, Phys. Rev. Lett. 87, 067202 (2001); Y. Zhang, E. Demler, and S. Sachdev, Competing orders in a magnetic field: Spin and charge order in the cuprate superconductors, Phys. Rev. B 66, 094501 (2002).

21. B. Lake, G. Aeppli, K. N. Clausen, D. F. McMorrow, K. Lefmann, N. E. Hussey, N. Mangkorntong, M. Nohara, H. Takagi, T. E. Mason, and A. Schröder, Spins in the Vortices of a HighTemperature Superconductor, Science 291, 1759 (2001).

22. B. Lake, H. M. Rønnow, N. B. Christensen, G. Aeppli, K. Lefmann, D. F. McMorrow, P. Vorderwisch, P. Smeibidl, N. Mangkorntong, T. Sasagawa, M. Nohara, H. Takagi, and T. E. Mason, Antiferromagnetic order induced by an applied magnetic field in a hightemperature superconductor, Nature 415, 299 (2002).

23. B. Khaykovich, S. Wakimoto, R. J. Birgeneau, M. A. Kastner, Y. S. Lee, P. Smeibidl, $\mathrm{P}$. Vorderwisch, and K. Yamada, Field-induced transition between magnetically disordered and ordered phases in underdoped $\mathrm{La}_{2-x} \mathrm{Sr}_{x} \mathrm{CuO}_{4}$, Phys. Rev. B 71, 220508 (2005).

24. J. Chang, Ch. Niedermayer, R. Gilardi, N. B. Christensen, H. M. Rønnow, D. F. McMorrow, M. Ay, J. Stahn, O. Sobolev, A. Hiess, S. Pailhes, C. Baines, N. Momono, M. Oda, M. Ido, and J. Mesot, Tuning competing orders in $\mathrm{La}_{2}{ }_{-x} \mathrm{Sr}_{x} \mathrm{CuO}_{4}$ cuprate superconductors by the application of an external magnetic field, Phys. Rev. B 78, 104525 (2008).

25. J. Chang, N. B. Christensen, Ch. Niedermayer, K. Lefmann, H. M. Rønnow, D. F. McMorrow, A. Schneidewind, P. Link, A. Hiess, M. Boehm, R. Mottl, S. Pailhes, N. Momono, M. Oda, M. Ido, and J. Mesot, Magnetic-Field-Induced Soft-Mode Quantum Phase Transition in the High-Temperature Superconductor $\mathrm{La}_{1.855} \mathrm{Sr}_{0.145} \mathrm{CuO}_{4}$ : An Inelastic NeutronScattering Study, Phys. Rev. Lett. 102, 177006 (2009).

26. D. Haug, V. Hinkov, A. Suchaneck, D. S. Inosov, N. B. Christensen, Ch. Niedermayer, P. Bourges, Y. Sidis, J. T. Park, A. Ivanov, C. T. Lin, J. Mesot, and B. Keimer, MagneticField-Enhanced Incommensurate Magnetic Order in the Underdoped High-Temperature Superconductor $\mathrm{YBa}_{2} \mathrm{Cu}_{3} \mathrm{O}_{6.45}$, Phys. Rev. Lett. 103, 017001 (2009).

27. E. M. Motoyama, G. Yu, I. M. Vishik, O. P. Vajk, P. K. Mang, and M. Greven, Spin correlations in the electron-doped high-transition-temperature superconductor $\mathrm{Nd}_{2-x} \mathrm{Ce}_{x} \mathrm{CuO} \mathrm{O}_{4 \pm \delta}$, Nature 445, 186 (2007).

28. R. K. Kaul, M. A. Metlitski, S. Sachdev, and C. Xu, Destruction of Néel order in the cuprates by electron doping, Phys. Rev. B 78, 045110 (2008).

29. Y. Kohsaka, C. Taylor, K. Fujita, A. Schmidt, C. Lupien, T. Hanaguri, M. Azuma, M. Takano, H. Eisaki, H. Takagi, S. Uchida, and J. C. Davis, An Intrinsic Bond-Centered Electronic Glass with Unidirectional Domains in Underdoped Cuprates, Science 315, 1380 (2007).

30. Y. Ando, K. Segawa, S. Komiya, and A. N. Lavrov, Electrical Resistivity Anisotropy from Self-Organized One Dimensionality in High-Temperature Superconductors, Phys. Rev. Lett. 88, 137005 (2002).

31. V. Hinkov, D. Haug, B. Fauqué, P. Bourges, Y. Sidis, A. Ivanov, C. Bernhard, C. T. Lin, and B. Keimer, Electronic Liquid Crystal State in the High-Temperature Superconductor $\mathrm{YBa}_{2} \mathrm{Cu}_{3} \mathrm{O}_{6.45}$, Science 319, 597 (2008).

32. G. Knebel, D. Aoki, and J. Flouquet, Magnetism and Superconductivity in CeRhIn arXiv:0911.5223 
33. N. Ni, M. E. Tillman, J.-Q. Yan, A. Kracher, S. T. Hannahs, S. L. Bud'ko, and P. C. Canfield, Effects of Co substitution on thermodynamic and transport properties and anisotropic $H_{c 2}$ in $\mathrm{Ba}\left(\mathrm{Fe}_{1-x} \mathrm{C}_{x}\right)_{2} \mathrm{As}_{2}$ single crystals, Phys. Rev. B 78, 214515 (2008).

34. S. Nandi, M. G. Kim, A. Kreyssig, R. M. Fernandes, D. K. Pratt, A. Thaler, N. Ni, S. L. Bud'ko, P. C. Canfield, J. Schmalian, R. J. McQueeney, and A. I. Goldman, Anomalous Suppression of the Orthorhombic Lattice Distortion in Superconducting $\mathrm{Ba}\left(\mathrm{Fe}_{1-x} \mathrm{Co}_{x}\right)_{2} \mathrm{As}_{2}$ Single Crystals, Phys. Rev. Lett. 104, 057006 (2010).

35. R. M. Fernandes, D. K. Pratt, W. Tian, J. Zarestky, A. Kreyssig, S. Nandi, M. G. Kim, A. Thaler, N. Ni, S. L. Bud'ko, P. C. Canfield, R. J. McQueeney, J. Schmalian, and A. I. Goldman, Unconventional pairing in the iron arsenide superconductors, Phys. Rev. B 81, 140501(R) (2010).

36. D. J. Scalapino, A Common Thread, Physica C 470, S1 (2010).

37. S. Sachdev, Exotic phases and quantum phase transitions: model systems and experiments, 24th Solvay Conference on Physics, Quantum Theory of Condensed Matter, Brussels, Oct 11-13, 2008, arXiv:0901.4103

38. S. Sachdev, Quantum Antiferromagnets in Two Dimensions in Low dimensional quantum field theories for condensed matter physicists, Yu Lu, S. Lundqvist, and G. Morandi eds., World Scientific, Singapore (1995); cond-mat/9303014

39. S. Sachdev, Quantum phases and phase transitions of Mott insulators in Quantum magnetism, U. Schollwöck, J. Richter, D. J. J. Farnell, and R. F. Bishop eds, Lecture Notes in Physics 645, Springer, Berlin (2004); cond-mat/0401041

40. S. Sachdev, Quantum phase transitions of correlated electrons in two dimensions, Lectures at the International Summer School on Fundamental Problems in Statistical Physics X, AugustSeptember 2001, Altenberg, Germany, Physica A 313, 252 (2002); cond-mat/0109419

41. M. P. Gelfand, R. R. P. Singh, and D. A. Huse, Zero-temperature ordering in two-dimensional frustrated quantum Heisenberg antiferromagnets, Phys. Rev. B 40, 10801 (1989).

42. A. Oosawa, M. Fujisawa, T. Osakabe, K. Kakurai, and H. Tanaka, Neutron Diffraction Study of the Pressure-Induced Magnetic Ordering in the Spin Gap System $\mathrm{TlCuCl}_{3}$, J. Phys. Soc. Jpn. 72, 1026 (2003).

43. Ch. Rüegg, N. Cavadini, A. Furrer, H.-U. Güdel, K. Krämer, H. Mutka, A. Wildes, K. Habicht, and $\mathrm{P}$. Vorderwisch, Bose-Einstein condensation of the triplet states in the magnetic insulator $\mathrm{TlCuCl}_{3}$, Nature 423, 62 (2003).

44. Ch. Rüegg, B. Normand, M. Matsumoto, A. Furrer, D. F. McMorrow, K. W. Krämer, H. U. Güdel, S. N. Gvasaliya, H. Mutka, and M. Boehm, Quantum Magnets under Pressure: Controlling Elementary Excitations in $\mathrm{TlCuCl}_{3}$ Phys. Rev. Lett. 100, 205701 (2008).

45. J. Callaway, Quantum Theory of the Solid State, Academic Press, New York (1974).

46. M. Matsumoto, C. Yasuda, S. Todo, and H. Takayama, Ground-state phase diagram of quantum Heisenberg antiferromagnets on the anisotropic dimerized square lattice, Phys. Rev. B 65, 014407 (2002).

47. S. Sachdev and R. N. Bhatt, Bond-operator representation of quantum spins: Mean-field theory of frustrated quantum Heisenberg antiferromagnets, Phys. Rev. B 41, 9323 (1990).

48. A. V. Chubukov and Th. Jolicoeur, Dimer stability region in a frustrated quantum Heisenberg antiferromagnet, Phys. Rev. B 44, 12050 (1991).

49. T. Sommer, M. Vojta, and K. W. Becker, Magnetic properties and spin waves of bilayer magnets in a uniform field, Eur. Phys. J. B 23, 329 (2001).

50. B. Normand and T. M. Rice, Dynamical properties of an antiferromagnet near the quantum critical point: Application to $\mathrm{LaCuO}_{2.5}$ Phys. Rev. B 56, 8760 (1997).

51. S. Sachdev, Theory of finite-temperature crossovers near quantum critical points close to,or above, their upper-critical dimension, Phys. Rev. B 55, 142 (1997).

52. D. Carpentier and L. Balents, Field theory for generalized Shastry-Sutherland models, Phys. Rev. B 65, 024427 (2002).

53. P. Calabrese, P. Parruccini, A. Pelissetto, and E. Vicari, Critical behavior of $O(2) \otimes O(N)$ symmetric models, Phys. Rev. B 70, 174439 (2004).

54. N. Read and S. Sachdev, Large- $N$ expansion for frustrated quantum antiferromagnets, Phys. Rev. Lett. 66, 1773 (1991). 
55. X. G. Wen, Mean-field theory of spin-liquid states with finite energy gap and topological orders, Phys. Rev. B 44, 2664 (1991).

56. S. Sachdev, Kagomé- and triangular-lattice Heisenberg antiferromagnets: Ordering from quantum fluctuations and quantum-disordered ground states with unconfined bosonic spinons, Phys. Rev. B 45, 12377 (1992).

57. F. Wang and A. Vishwanath, Spin-liquid states on the triangular and Kagom lattices: A projective-symmetry-group analysis of Schwinger boson states, Phys. Rev. B 74, 174423 (2006).

58. D. P. Arovas and A. Auerbach, Functional integral theories of low-dimensional quantum Heisenberg models, Phys. Rev. B 38, 316 (1988); Spin Dynamics in the Square-Lattice Antiferromagnet, Phys. Rev. Lett. 61, 617 (1988).

59. N. Read and S. Sachdev, Some features of the phase diagram of the square lattice $S U(N)$ antiferromagnet, Nucl. Phys. B 316, 609 (1989).

60. N. Read and S. Sachdev, Valence-bond and spin-Peierls ground states of low-dimensional quantum antiferromagnets, Phys. Rev. Lett. 62, 1694 (1989).

61. N. Read and S. Sachdev, Spin-Peierls, valence-bond solid, and Néel ground states of lowdimensional quantum antiferromagnets, Phys. Rev. B 42, 4568 (1990).

62. S. Sachdev and N. Read, Large $N$ expansion for frustrated and doped quantum antiferromagnets, Int. J. Mod. Phys. B 5, 219 (1991); cond-mat/0402109

63. I. Affleck, The quantum Hall effects, $\sigma$-models at $\Theta=\pi$ and quantum spin chains, Nucl. Phys. B 257, 397 (1985); Exact critical exponents for quantum spin chains, non-linear $\sigma$ models at $\theta=\pi$ and the quantum hall effect, Nucl. Phys. B 265, 409 (1985).

64. T. Einarsson and H. Johannesson, Effective-action approach to the frustrated Heisenberg antiferromagnet in two dimensions, Phys. Rev. B 43, 5867 (1991); T. Einarsson, P. Frojdh, and $\mathrm{H}$. Johannesson, Weakly frustrated spin-1/2 Heisenberg antiferromagnet in two dimensions: Thermodynamic parameters and the stability of the Néel state, Phys. Rev. 45, 13121 (1992).

65. P. Chandra, P. Coleman, and A. I. Larkin, Ising transition in frustrated Heisenberg models, Phys. Rev. Lett. 64, 88 (1990); P. Chandra and P. Coleman, Twisted magnets and twisted superfluids, Int. J. Mod. Phys. B 3, 1729 (1989).

66. B. I. Halperin and W. M. Saslow, Hydrodynamic theory of spin waves in spin glasses and other systems with noncollinear spin orientations, Phys. Rev. B 16, 2154 (1977).

67. T. Dombre and N. Read, Nonlinear $\sigma$ models for triangular quantum antiferromagnets, Phys. Rev. B 39, 6797 (1989).

68. P. Azaria, B. Delamotte, and T. Jolicoeur, Nonuniversality in helical and canted-spin systems, Phys. Rev. Lett. 64, 3175 (1990)

69. I. Affleck and J. B. Marston, Large-n limit of the Heisenberg-Hubbard model: Implications for high- $T_{c}$ superconductors, Phys. Rev. B37, 3774 (1988).

70. D. Rokhsar and S. Kivelson, Superconductivity and the Quantum Hard-Core Dimer Gas, Phys. Rev. Lett. 61, 2376 (1988).

71. C. Henley, Ordering due to disorder in a frustrated vector antiferromagnet, Phys. Rev. Lett. 62, 2056 (1989).

72. A. Chubukov, First-order transition in frustrated quantum antiferromagnets, Phys. Rev. B 44, 392 (1991).

73. F. Mila, D. Poilbanc, and C. Bruder, Spin dynamics in a frustrated magnet with short-range order, Phys. Rev. B 43, 7891 (1991).

74. J. Stephenson, Range of order in antiferromagnets with next-nearest neighbor coupling, Can. J. Phys. 48, 2118, 1724 (1970).

75. A. M. Polyakov, Gauge Fields and Strings, Harwood, New York (1987).

76. A. M. Polyakov, Quark confinement and topology of gauge theories, Nucl. Phys. B 120, 429 (1977).

77. T. Senthil, A. Vishwanath, L. Balents, S. Sachdev, and M. P. A. Fisher, Deconfined Quantum Critical Points, Science 303, 1490 (2004).

78. T. Senthil, L. Balents, S. Sachdev, A. Vishwanath, and M. P. A. Fisher, Quantum criticality beyond the Landau-Ginzburg-Wilson paradigm, Phys. Rev. B 70, 144407 (2004). 
79. E. Fradkin and S. H. Shenker, Phase diagrams of lattice gauge theories with Higgs fields, Phys. Rev. D 19, 3682 (1979).

80. T. Senthil and M. P. A. Fisher, $Z_{2}$ gauge theory of electron fractionalization in strongly correlated systems, Phys. Rev. B 62, 7850 (2000).

81. A. Y. Kitaev, Fault-tolerant quantum computation by anyons, Annals of Physics 303, 2 (2003).

82. C. Xu and S. Sachdev, Global phase diagrams of frustrated quantum antiferromagnets in two dimensions: Doubled Chern-Simons theory, Phys. Rev. B 79, 064405 (2009).

83. Y. Qi, C. Xu, and S. Sachdev, Dynamics and transport of the $Z_{2}$ spin liquid: application to $\kappa-(E T)_{2} \mathrm{Cu}_{2}(C N)_{3}$, Phys. Rev. Lett. 102, 176401 (2009).

84. I. F. Herbut, V. Juričić, and B. Roy, Theory of interacting electrons on the honeycomb lattice, Phys. Rev. B 79, 085116 (2009).

85. V. Juričić, I. F. Herbut, and G. W. Semenoff, Coulomb interaction at the metal-insulator critical point in graphene, Phys. Rev. B 80, 081405(R) (2009).

86. M. De Prato, A. Pelissetto, and E. Vicari, Spin-density-wave order in cuprates, Phys. Rev. B 74, 144507 (2006).

87. A. Pelissetto, S. Sachdev and E. Vicari, Nodal Quasiparticles and the Onset of Spin-DensityWave Order in Cuprate Superconductors, Phys. Rev. Lett. 101, 027005 (2008).

88. E.-A. Kim, M. J. Lawler, P. Oreto, S. Sachdev, E. Fradkin, and S. A. Kivelson, Theory of the nodal nematic quantum phase transition in superconductors, Phys. Rev. B 77, 184514 (2008).

89. M. Vojta, Y. Zhang, and S. Sachdev, Quantum Phase Transitions in d-Wave Superconductors, Phys. Rev. Lett. 85, 4940 (2000); 100, 089904(E) (2008).

90. M. Vojta, Y. Zhang, and S. Sachdev, Renormalization group analysis of quantum critical points in d-wave superconductors, Int. J. Mod. Phys. B 14, 3719 (2000).

91. R. B. Laughlin, Magnetic Induction of $d_{x^{2}-y^{2}}+i d_{x y}$ Order in High-T $T_{c}$ Superconductors, Phys. Rev. Lett. 80, 5188 (1998).

92. M.-R. Li, P. J. Hirschfeld, and P. Woelfle, Vortex state of a d-wave superconductor at low temperatures, Phys. Rev. B 63, 054504 (2001).

93. D. V. Khveshchenko and J. Paaske, Incipient Nodal Pairing in Planar d-wave Superconductors, Phys. Rev. Lett. 86, 4672 (2001).

94. B. Rosenstein, B. J. Warr, and S. H. Park, Dynamical symmetry breaking in four-fermion interaction models, Phys. Rep. 205, 59 (1991).

95. L. Kärkkäinen, R. Lacaze, P. Lacock, and B. Petersson, Critical behaviour of the threedimensional Gross-Neveu and Higgs-Yukawa models, Nucl. Phys. B 415, 781 (1994).

96. Y. Huh and S. Sachdev, Renormalization group theory of nematic ordering in d-wave superconductors, Phys. Rev. B 78, 064512 (2008).

97. L. Fritz and S. Sachdev, Signatures of the nematic ordering transitions in the thermal conductivity of d-wave superconductors, Phys. Rev. B 80, 144503 (2009).

98. M. A. Metlitksi and S. Sachdev, Quantum phase transitions of metals in two spatial dimensions: I. Ising-nematic order, Phys. Rev. B 82, 075127 (2010).

99. M. A. Metlitski and S. Sachdev, Quantum phase transitions of metals in two spatial dimensions: II. Spin density wave order,Phys. Rev. B 82, 075128 (2010).

100. H. v. Löhneysen, A. Rosch, M. Vojta, and P. Wölfle, Fermi-liquid instabilities at magnetic quantum phase transitions, Rev. Mod. Phys. 79, 1015 (2007).

101. M. A. Metlitski and S. Sachdev, Instabilities near the onset of spin density wave order in metals, New Journal of Physics 12, 105007 (2010).

102. S. Sachdev, Condensed matter and AdS/CFT, arXiv:1002.2947 